\title{
Optimal Power Flow Considering Intermittent Solar and Wind Generation using Multi-Operator Differential Evolution Algorithm
}

\author{
Karam M. Sallam, ${ }^{\mathrm{a}, *}$ Md Alamgir Hossain ${ }^{\mathrm{b}}$, Seham S. Elsayed ${ }^{\mathrm{b}}$, Ripon K. Chakrabortty ${ }^{\mathrm{b}}$, Michael J. Ryan ${ }^{\mathrm{b}}$, \\ Mohammad A. Abidoc \\ ${ }^{a}$ The Faculty of Computers $\mathcal{F}$ Information, Zagazig University, Egypt \\ ${ }^{b}$ Capability Systems Centre, School of Eng. $\mathcal{E}$ IT, UNSW Canberra at ADFA, Australia \\ ${ }^{c}$ Electrical Engineering Department, King Fahd University of Petroleum E Mineralitc, Dhahran, Saudi Arabia
}

\begin{abstract}
In this paper, a multi-operator differential evolution algorithm (MODE) is proposed to solve the Optimal Power Flow (OPF) problem and is called MODE-OPF. The MODE-OPF utilizes the strengths of more than one differential evolution (DE) operator in a single algorithmic framework. Additionally, an adaptive method (AM) is proposed to update the number of solutions evolved by each DE operator based on both the diversity of population and quality of solutions. This adaptive method has the ability to maintain diversity at the early stages of the optimization process and boost convergence at the later ones. The performance of the proposed MODE-OPF is tested by solving OPF problems for both small and large IEEE bus systems (i.e., IEEE-30 and IEEE-118) while considering the intermittent solar and wind power generation. To prove the suitability of this proposed algorithm, its performance has been compared against several state-of-the-art optimization algorithms, where MODE-OPF outperforms other algorithms in all experimental results and thereby improving a network's performance with lower cost. MODE-OPF decreases the total generation cost up to $24.08 \%$, the real power loss up to $6.80 \%$ and the total generation cost with emission up to $8.56 \%$.
\end{abstract}

Keywords: Evolutionary Algorithms, differential evolution, constraints handling techniques, optimal power flow, renewable energy

\section{Introduction}

The complexity of electric power system is gradually increasing due to a number of reasons, including high power demand and intermittent power generation from renewable energy sources (RESs), in addition to its well-known large scale, several dynamic/static states and difficult interfaces among components. Effective management and control of such a system are crucial to maintain a reliable, secure power supply [1]. However, it is one of the most challenging tasks for the system operators because of the close monitoring required of the large range of data that need to be measured, computed and controlled with appropriate actions [2]. To manage the utility grid effectively and efficiently, optimal power flow (OPF) and economic dispatch are generally performed by the network operators [3]. Economic dispatch deals with the least-cost generation dispatch to fulfil the power demand, but it ignores power flow constraints that may violate the physical limits of a transmission network.

The OPF problem, one of the fundamental tools to maintain reliable, economic power system operation, has drawn the attention of researchers since it was introduced by Carpentier in 1962 [4]. The problem is to optimize a chosen objective function, such as total generation cost and active power loss, through the appropriate selection of the control variables while fulfilling many inequality and equality constraints, such as power flow equations, and the bounds of control and dependent variables. The control variables, including real powers and bus voltage of generators,

\footnotetext{
${ }^{*}$ I am corresponding author

Email addresses: Karam_sallam@zu.edu.eg (Karam M. Sallam ), Md.Hossain6@unsw.edu.au (Md Alamgir Hossain), seham.elsayed@adfa.edu.au (Seham S. Elsayed), r. chakrabortty@adfa.edu.au (Ripon K. Chakrabortty), m.ryan@adfa.edu.au (Michael J. Ryan), mabido@kfupm.edu.sa (Mohammad A. Abido)
} 
switchable reactive power of Volt-Amps-Reactive (VAR), and tap settings of transformers, are chosen in a way that minimizes system losses and maintains dependent variables (generator reactive power, load bus voltage and network line flow) within acceptable ranges [5]. As the OPF problem has many control variables that are not all continuous variables, such as the outputs of VAR that are discrete variables, it is a non-convex, non-linear, multi-modal, and largescale constrained optimization problem [6]. Therefore, solving it is a challenging task in power system optimization and is of current interest to many power companies.

There are a number of solution approaches in the literature that deals with the OPF problems. One approach involves mathematical techniques that are widely utilized to determine the optimal values of all control variables in power system operation. The mathematical approach, including linear programming [7], nonlinear programming [8], quadratic programming [9], interior point method [10] and Newton-based methods [11], provides the optimal solution of OPF problems; however, several drawbacks of this approach are also inevitable. For example, some of the noticeable shortcomings of existing mathematical approaches include no guarantee of accuracy for the linear program, poor convergence and algorithm complication for the non-linear programming, the sensitivity of initial guess for the Newton-based algorithm, solving complexities aroused for non-linear and quadratic objective functions in the interior point algorithm [12]. In addition, mathematical approaches have issues in finding global optima (i.e., trapping in local optima) and specific objective function restrictions, such as prohibited operating zones, valve point effects and piece-wise quadratic cost function $[3,13]$. It is noteworthy that power system operators require a more robust, faster OPF solver to reach an effective decision for the economic operation of a network. Therefore, it becomes important to propose and develop effective optimization algorithms that can address the issues of traditional mathematical or exact approaches.

In recent decades, many evolutionary-based optimization algorithms have been developed to successfully find the optimal solution of complex OPF problems. Abido [5] developed a particle swarm optimization (PSO) algorithm to solve the OPF problem of several objective functions with mild constraints, such as minimization of fuel cost and voltage profile. In [14], an enhanced genetic algorithm (GA) was presented to solve the OPF with continuous (active power output) and discrete (transformer tap setting) control variables. The problem-specific operators were included to boost the efficiency of the algorithm. Teaching-learning based optimization (TLBO) algorithm was applied in [15] to find the optimal solution for OPF problems. An enhanced artificial bee colony (ABC) algorithm based on orthogonal learning was presented in [16]to handle the OPF problem. The performance of the algorithm was boosted by maintaining a balance between the exploration and exploitation search. In [17], a hybrid algorithm based on PSO and gravitational search algorithms was presented. An improved colliding bodies optimization (ICBO) algorithm was developed in [18] to solve the OPF problem and the performance of the ICBO was compared with the CBO and other well-known approaches. A modified Sine-Cosine technique with a Lévy flight was integrated to improve the exploration of the algorithm [19]. In [20], a moth swarm algorithm to solve the OPF problem was presented, where an adaptive crossover with mutation scaled by Lévy flights was used for maintaining solutions diversity. An enhanced social spider optimization algorithm was developed in [21] to individually optimize fuel cost, voltage deviation, power loss and polluted emission. The algorithm was improved by adding three modifications: changing the movement strategy of male and female spiders, and fixing the female spider rate. A hybrid approach of moth swarm algorithm and gravitational search algorithm was developed in [22] to efficiently solve the OPF problem. A black hole algorithm was used in [15] to solve the OPF problem. Despite some success, there are still a number of shortcomings in these approaches, such as the problem formulations in unconstrained optimization that may lead to violation of constraints, in addition to the improving performance of algorithms for solving the problem with the intermittent nature of renewable energy sources.

The differential evolutionary (DE) algorithm developed by Storn and Price in 1997 [23] has good convergence, robustness and the ability to obtain the global optimal. Consequently, DE has achieved better results in solving benchmark problems as compared to other algorithms and it has been applied in many fields to solve complex problems [24]. DE and DE variants (success-history based adaptive DE) are also successfully applied in the power systems to solve the complex problem of OPF. In [25], a parallel DE algorithm was presented that uses small-population for solving short-term hydro-thermal scheduling problem with power flow constraints. A DE algorithm to solve OPF problem was employed in [26], who also evaluated the performance of proper constraint handling techniques — self-adaptive penalty (SP), the superiority of feasibly solutions and an ensemble of these two constraint handling techniques. In [27], the grey wolf optimizer (GWO) and DE algorithms were developed to find the optimal solution of the OPF problem. Hybrid DE and harmony search (HS) algorithms were developed in [28] who replaced operation of the evolution 
algorithm by a pitch adjustment operation and then a harmony algorithm was used to improve the global convergence. An enhanced adaptive DE algorithm that considers SP constraint handling technique was also developed in [12] to determine the near-optimal solution of the OPF problem. The performance of the algorithm was enhanced using four operators: crossover rate $(\mathrm{Cr})$ sorting mechanism, re-randomizing $\mathrm{Cr}$ and scale factor $(F)$, dynamic population reduction strategy, and $\mathrm{SP}$ constraint linear technique.

Although several optimization algorithms and DE algorithms have been introduced to find the optimal or nearoptimal solution of optimization problems, especially OPF problems in the literature, no single evolutionary algorithm (EA) or search operator has consistently been the best solution to all of the problems [29, 30, 31]. As a result, many researchers and practitioners proposed several innovations that use multiple EAs in a single algorithmic system, called multi-methods or multi-operators, to satisfy this constraint [32, 33, 29]. Nevertheless, there is still scope for progress in their efficiency by developing an effective algorithm for a better, faster and reliable solution of the complex problem. This encourages us to develop an effective advanced multi-operator DE variant, called MODE-OPF, that uses many DE mutation operators in a single algorithmic system to solve OPF. In order to emphasize the most effective DE mutation strategy during the optimization process, an adaptive method (AM) process that uses both the diversity of the population and the quality of solutions has been proposed. We design this AM in a way that is capable of managing the diversification and intensification properties throughout the optimization process. Additionally, instead of considering all constraints to calculate the constraints violation, MODE-OPF starts with a number of them and then gradually increases the number of constraints until satisfying all of them.

MODE-OPF starts with a random initial population of NP solutions. Then, the fitness function value and the total constraint violation of each individual is computed with the number of current objective function evaluation. The whole population is evolved by a number of DE mutation strategies, (i.e., each DE operator produces equal number of new solutions). The diversity for the solutions generated by each DE operators and the quality of solutions are computed and recorded. Based on these two criteria and at the end of each iteration, the number of solutions evolved by each DE operator are updated as described in Subsection 3.2, with a minimum number of solutions are preserved. This process lasts until the maximum number of fitness evaluation exhausted. Five different objective functions (consequently referred as cases) are considered to demonstrate the efficacy of the proposed MODE-OPF approach. Numerous experiments are performed for modified IEEE 30-bus system and IEEE 118-bus system. The efficacy of this proposed approach is validated by a rigorous comparison against different state-of-the-art algorithms for those IEEE bus systems.

The major contributions of this paper are as follows:

1. A multi-operator DE (MODE) has been proposed in order to solve the optimal power flow (OPF) optimization problems.

2. An adaptive method (AM) has been developed, based on both the diversity of the solutions generated by each DE operators and the quality of solutions. This AM favors diversity at the early stages and quality at the later stages of the evolutionary process. Based on this AM the number of solutions evolved by each DE operator is updated.

3. This paper employs a unique way of handling constraints different from conventional ones to improve its performance, i.e., the proposed approach starts with a subset of the constraints to satisfy, and then gradually adds another subset until all constraints are satisfied.

4. To demonstrate the effectiveness of the algorithm proposed, a modified IEEE 30-bus and an IEEE 118-bus networks are used, where intermittent wind and solar power generation are modeled to the problem formulation using different probability distribution functions.

The rest of the paper is organized as follows. Section 2 introduces the OPF mathematical formulation. The proposed algorithm and its components are given in Section 3. Section 4 introduces the experimental results and comparison with rival algorithms. Conclusions and some future works are presented in Section 5.

\section{Mathematical model and constraints of OPF}

The target of the OPF problem is to operate a power system effectively and efficiently by optimizing control variables by considering either one or multiple objective functions after maintaining physical and thermal limits of the 
network. Mathematically, the OPF problem can be formulated as a non-linear constrained optimization problem as follows:

Minimize $F(x, u)$

Subject to $g(x, u) \leq 0$

and $h(x, u)=0$

where $u$ is a vector of independent or control variables; $x$ is a vector of dependent or state variables; $F(x, u)$ is the objective function; $g(x, u)$ is the inequality constraints and $h(x, u)$ is the equality constraints. The control variables, $u$, and the state variables, $x$, in power system are defined in the following subsection.

\subsection{Control variables}

There is a set of variables that are adjusted by conducting an optimization algorithm to achieve optimal operation of the power system, termed as control or independent variables. They are optimally set by solving the OPF problem using optimization algorithms in order to maintain a reliable, secure and economic power supply to consumers. In our study, eleven control variables are considered to optimally solve the OPF problems for improving the network's performance with minimum operational cost. The control variables include the power settings of all the generators (except the swing generator) and the bus voltages of all the generators. Without losing generality, this paper uses fixed tap settings of all transformers at $1 \mathrm{p}$.u to create the same facility of analysis with the existing literature [12, 26, 34, 35]. The set of control variables can be expressed as follows:

$$
u^{T}=\left[P_{G_{2}} \ldots P_{G_{N G}}, V_{G_{1}} \ldots V_{G_{N G}}\right]
$$

where $N G$ is the number of generators; $P_{G}$ is the active power generation, including solar and wind generators, at PV buses (except the slack bus); $V_{G}$ is the voltage magnitude of generators at PV buses, including solar and wind generators.

\subsection{State variables}

The state variables are the set of variables that represent the unique state of a power system. The state variables are dependent on the control variables. Mathematically, the state variables can be expressed in vector form as follows:

$$
x^{T}=\left[P_{G_{1}}, V_{L_{1}} \ldots V_{L_{N L}}, Q_{G_{1}} \ldots Q_{G_{N G}}, S_{l_{1}} \ldots S_{l_{n l}}\right]
$$

where $P_{G_{1}}$ is the active power at the slack bus; $V_{L}$ is the voltage magnitude at PQ buses (load buses); $Q_{G}$ is the reactive power generation of generating units and $S_{l}$ is the transmission line loadings (or line flow). $N L, N G$ and $n l$ are the number of load buses, the number of generators and the number of transmission lines, respectively.

\subsection{Objective functions}

The target of the optimization problem is to solve a chosen function while maintaining the thermal and physical constraints of a power system. In this study, we have minimized five single objective functions to demonstrate the performance of the proposed EA. Minimizing each objective function has its own purposes to optimize parameters, as illustrated in Figure 1. The mathematical problem formulations of objective functions are provided in the following subsection.

\subsubsection{Case 1: Operating cost}

The operating cost of generators generally depends on running cost (i.e., the fuel cost of thermal generators), but in the case of wind and solar generators, there is virtually no operating cost, except some of the factors that need to be considered for reducing the payback period. It is well-known that renewable powers are intermittent in nature, leading to complexity to fulfil the power supply committed. The deficit in total output power must be compensated by a spinning reserve. The problem formulations of operating costs are presented below. 


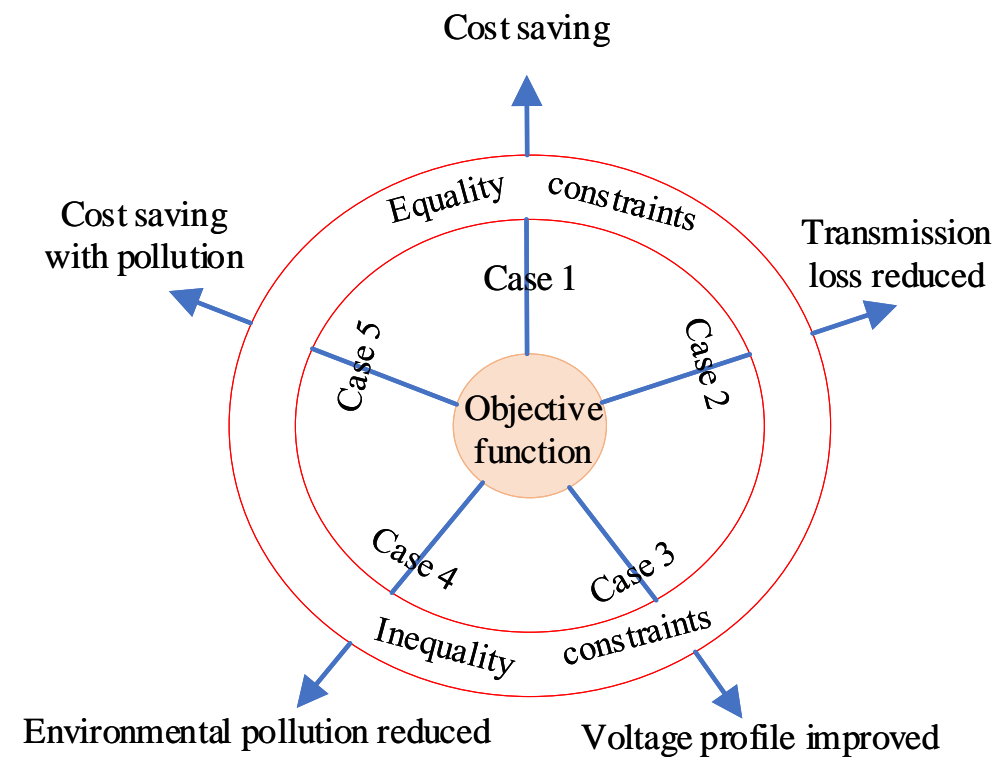

Figure 1: Objective functions of OPF problems.

Thermal Generators: Achieving the minimum operating cost of generating units is the main goal of economic power dispatch problem. Each generating unit has its own operating cost represented by a quadratic function. The cost function of generators in terms of cost per hour ( $\$ / \mathrm{h})$ for generating power (in MW) can be expressed as follows:

$$
F=\sum_{i=1}^{N G}\left[a_{i}+b_{i} P_{G i}+c_{i} P_{G i}^{2}\right]
$$

where $a_{i}, b_{i}$ and $c_{i}$ are the cost coefficients of the $i$ th generating unit.

For realistic and precise modeling of fuel cost, the valve-point effect (a ripple like effect on fuel cost as shown in Figure 2) of generating units need to be included in the cost function. This signifies the fuel cost of the units is not continuous but non-linear. The incremental fuel cost of the generation units with value-point loading is represented as follows [12]:

$$
F_{C}=\sum_{i=1}^{N G}\left[a_{i}+b_{i} P_{G i}+c_{i} P_{G i}^{2}+\left|d_{i} \times \sin \left(e_{i} \times\left(P_{G i}^{\min }-P_{G i}\right)\right)\right|\right]
$$

where $d_{i}$ and $e_{i}$ are the coefficients that represent the valve-point loading effect.

Wind generators: Although the operating cost of a wind generator can be considered to be zero, maintenance and renewal cost of wind power can be expressed as follows:

$$
C_{W T}=c_{w t} \times P_{W T}
$$

where $P_{W T}$ is the scheduled power committed to supply the network and $c_{w t}$ is the cost coefficient of wind generators. As power generation from wind farm is intermittent, it may not supply the power committed (i.e., overestimated power supply). Therefore, the cost of extra power supply from spinning reserve can be applied as follows: [34]:

$$
C_{R W T}=c_{r w t} \int_{0}^{P_{W T}}\left(P_{W T}-p_{w}\right) f_{w}\left(p_{w}\right) d p_{w}
$$

where $c_{r w t}$ and $p_{w}$ are the reserve cost for overestimating wind power and available wind power at that time. $P_{W T}$ is 


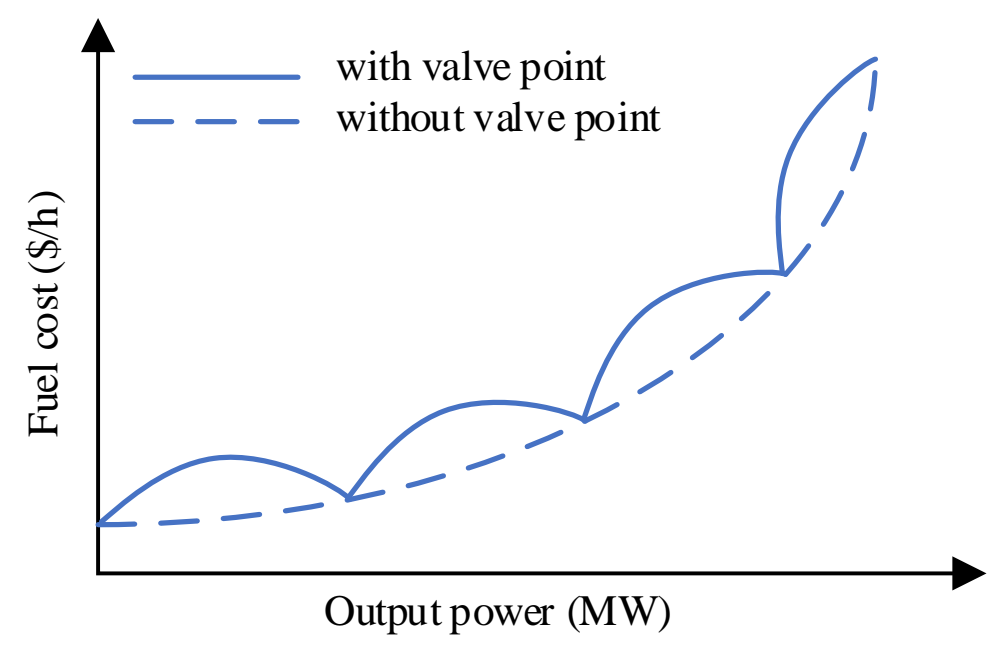

Figure 2: Objective function for a single generator with valve-point effect.

the scheduled wind power and $f_{w}\left(p_{w}\right)$ is the probability density function.

If available power generation is higher than the scheduled power committed, then extra power is generally wastedcalled the underestimate amount. This causes delay in the payback period; therefore, a penalty is imposed on underestimate amount as follows:

$$
C_{P W T}=c_{p w t} \int_{P_{W T}}^{P_{W R}}\left(p_{w}-P_{W T}\right) f_{w}\left(p_{w}\right) d p_{w}
$$

where $c_{p w t}$ and $P_{W R}$ are the penalty cost for underestimating wind power generation and the rated wind power output. The power output of wind generators can be expressed in terms of wind speed as follows [36]:

$$
p_{w}=\left\{\begin{array}{lr}
0 & \text { if } v_{f}<v \text { or } v<v_{c} \\
P_{R W T}\left(\frac{v^{3}-v_{c}^{3}}{v_{r}^{3}-v_{c}^{3}}\right) & \text { if } v_{c} \leq v \leq v_{r} \\
P_{R W T} & \text { if } v_{r}<v \leq v_{f}
\end{array}\right.
$$

where $v_{r}$ is the rated wind speed, $16 \mathrm{~m} / \mathrm{s} ; v_{c}$ refers to cut-in wind speed, $3 \mathrm{~m} / \mathrm{s} ; v_{f}$ is the cut-off wind speed $(25 \mathrm{~m} / \mathrm{s})$, and $v$ represents wind speed.

As the output power of wind generators are a mixture of discrete and continuous, its probability can be calculated as follows [35]:

$$
\begin{aligned}
f_{w}\left(p_{w}=0\right) & =1-e^{-\left(\frac{v_{c}}{\alpha}\right)^{\beta}}+e^{-\left(\frac{v_{f}}{\alpha}\right)^{\beta}} \\
f_{w}\left(p_{w}=P_{W R}\right) & =e^{-\left(\frac{v_{r}}{\alpha}\right)^{\beta}}+e^{-\left(\frac{v_{f}}{\alpha}\right)^{\beta}} \\
f_{w}\left(p_{w}\right) & =\frac{\beta\left(v_{r}-v_{c}\right)}{\alpha \beta P_{W R}}\left[v_{c}+\frac{p_{w}}{P_{W R}}\left(v_{r}-v_{c}\right)\right]^{\beta-1} e^{-\left(\frac{v_{c}+\frac{p_{w}}{P_{W R}}\left(v_{r}-v_{c}\right)}{\alpha}\right)^{\beta}}
\end{aligned}
$$

where $v_{c}, v_{f}$ and $v_{r}$ are the cut-in, cut-out and rated wind speed, respectively.

As wind speed distribution can be represented by Weibull probability density function (PDF), the probability of wind 
speed $v \mathrm{~m} / \mathrm{s}$ can be written as follows [37]:

$$
f_{v}=\left(\frac{\beta}{\alpha}\right)\left(\frac{v}{\alpha}\right)^{\beta-1} e^{-(v / \alpha)^{\beta}}
$$

where $\alpha$ is the Weibull scale parameter of PDF and $\beta$ is the Weibull shape parameter of PDF. Figure 3 and 4 represent the Weibull fitting and wind speed probability distribution by generating 8000 Monte-Carlo scenarios. The $\beta$ of Weibull shape is 2 for the both wind speed distributions, and $\alpha$ is 9 for wind speed of windfarm 1 and 10 for wind speed of windfarm 2 .

Solar generators: The cost of solar power generation $\left(C_{P S}\right)$ for maintenance, renewal and payback period can be determined by the following equation [38]:

$$
C_{P S}=c_{S c} \times P_{P S}
$$

where $c_{s c}$ and $P_{P S}$ are the solar cost coefficient and the scheduled power generation, respectively. For the overestimate of solar power generation, the reserve cost $\left(C_{r s}\right)$ can be incorporated as follows [38]:

$$
C_{R S}=c_{r s} * f_{s}\left(p_{s}<P_{P S}\right) *\left[P_{P S}-E\left(p_{s}<P_{P S}\right)\right]
$$

where $c_{r s}, p_{s}, f_{s}\left(p_{s}<P_{P S}\right)$ and $E\left(p_{s}<P_{P S}\right)$ are the reserve cost coefficient of overestimation, the available solar power generation, the probability of power shortage from scheduled generation, and the expectation of solar power generation, respectively.

The underestimate of the solar power generation, leading to power wastage, should be penalized and therefore it $\left(C_{P S}\right)$ can be formulated as follows:

$$
C_{P S}=c_{p s} * f_{s}\left(p_{s}>P_{P S}\right) *\left[E\left(p_{s}>P_{P S}\right)-P_{P S}\right]
$$

where $c_{p s}, f_{s}\left(p_{s}>P_{P S}\right)$ and $E\left(p_{s}>P_{P S}\right)$ are the penalty cost coefficient of underestimate in solar power generation, the probability of power generation exceeded from scheduled, and the expectation of the power higher than $P_{P S}$, respectively.

The probability of solar irradiance $\left(G_{s}\right)$ can be represented by the Lognormal PDF with mean, $\mu$, and standard deviation, $\sigma$, as follows [39]:

$$
f_{G}=\frac{1}{G_{s} \sigma \sqrt{2 \pi}} \mathrm{e}^{\left\{\frac{-\left(\ln G_{s}-\mu\right)^{2}}{2 \sigma^{2}}\right\}} \text { if } G_{s}>0
$$

Figure 5 demonstrates solar irradiation probability distribution and Lognormal fitting by applying 8000 scenarios of Monte Carlo simulation. For the Lognormal fitting, we have used $\mu=5$ and $\sigma=0.6$. Power generation from solar irradiation can be written as follows [40]:

$$
p_{s}=\left\{\begin{array}{lr}
P_{R S}\left(\frac{G_{s}^{2}}{G_{s d} R_{c}}\right) & \text { if } 0<G_{s}<R_{c} \\
P_{R S}\left(\frac{G_{s}}{G_{s d}}\right) & \text { otherwise }
\end{array}\right.
$$

where $G_{s d}$ and $R_{c}$ are the solar irradiance at standard value $\left(1000 \mathrm{~W} / \mathrm{m}^{2}\right)$, and a certain irradiance point set $(120$ $\mathrm{W} / \mathrm{m}^{2}$ ), respectively. $P_{R S}$ is the rated solar power generation.

Please note that the reserve cost of wind turbines and solar generators are different from one another due to their level of uncertainty involvement, government promotion on specific power generation and their individual payback period considered.

\subsubsection{Case 2: Real power loss}

As power flows through the transmission line, there is power loss during power transfer from generating stations to substations due to the existence of resistance across the line. The real power loss (in MW) can be reduced by 


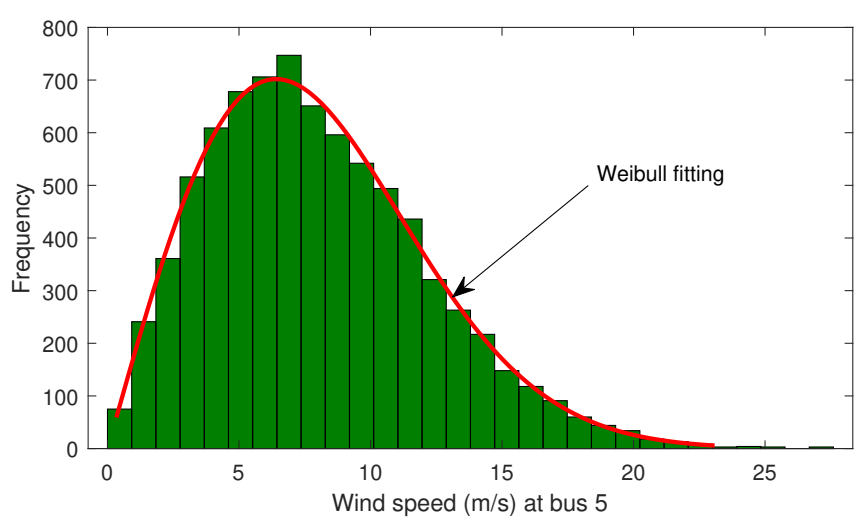

Figure 3: Wind power distribution at bus 5

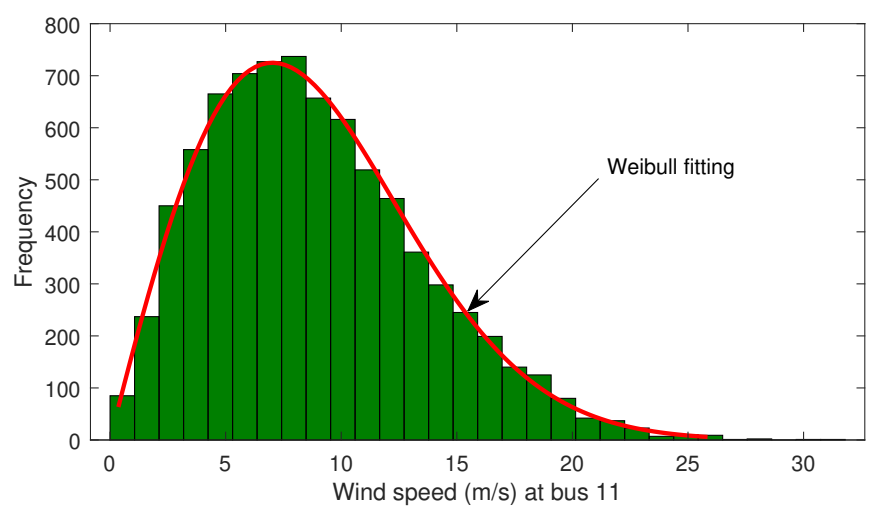

Figure 4: Wind power distribution at bus 11

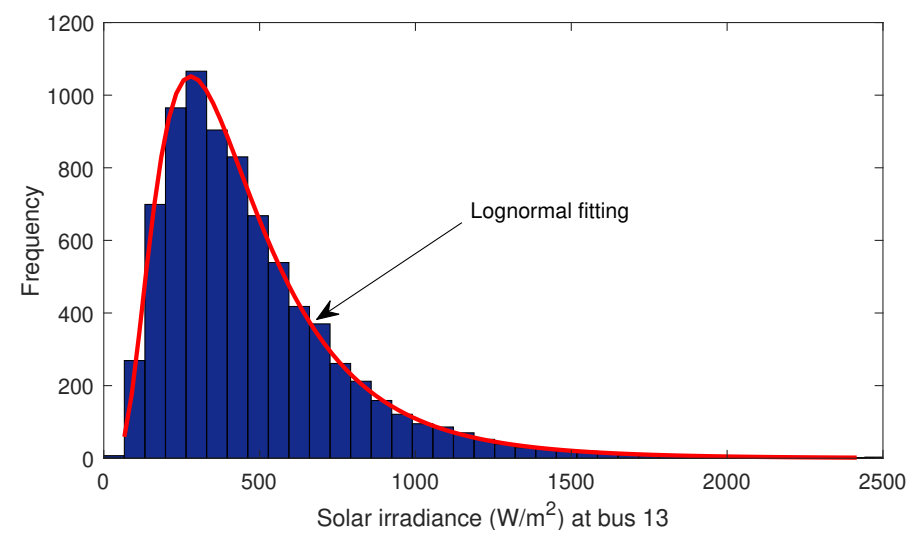

Figure 5: Solar irradiance distribution at bus 13 
formulating a cost function as follows:

$$
F_{\text {loss }}=\sum_{i=1}^{n l} \sum_{j \neq i}^{n l} G_{i j}\left[V_{i}^{2}+V_{j}^{2}-2 V_{i} V_{j} \cos \left(\delta_{i}-\delta_{j}\right)\right]
$$

where $G_{i j}$ is the conductance of a branch between $i$ and $j$ buses; $\delta_{i}$ and $\delta_{j}$ are the voltage angle at bus $i$ and $j$, respectively; $V_{i}$ and $V_{j}$ are the voltage magnitude at buses $i$ and $j$, respectively.

\subsubsection{Case 3: Voltage deviation}

The voltage profile of a network can be improved by defining an objective function in terms of voltage deviation at load buses. It is essential to maintain a voltage profile within predefined acceptable ranges to uphold the stability and security of the power system. The voltage profile deviation is expressed as the cumulative deviation of voltages of all the load buses from the reference voltage, 1 p.u. Therefore, the objective function for minimizing voltage deviation $\left(F_{V D}\right)$ can be expressed as follows:

$$
F_{V D}=\sum_{i=1}^{N L}\left|V_{L i}-V_{\text {ref }}\right|
$$

where $V_{\text {ref }}$ is the reference voltage that is preferable to maintained at the network.

\subsubsection{Case 4: Emission control}

As conventional power generating units emit harmful and greenhouse gases, such as $\mathrm{SO}_{x}, \mathrm{NO}_{x}$ and $\mathrm{CO}_{2}$, into the environment, there is a recent trend to impose a penalty for minimizing gas emission. The minimization of these gas in terms of tonnes per hour $(\mathrm{t} / \mathrm{h})$ can be described by the following objective function:

$$
F_{E}=\sum_{i=1}^{N G}\left[\alpha_{i}+\beta_{i} P_{G i}+\gamma_{i} P_{G i}^{2}+\omega_{i} e^{\mu_{i} P_{G i}}\right]
$$

where $\alpha_{i}, \beta_{i}, \gamma_{i}$ and $\omega_{i}$ are the emission coefficients corresponding to the $i$ th generator.

\subsubsection{Case 5: Operating cost with emission}

It is sometimes important to consider both the operating cost and greenhouse gas emission control simultaneously to promote renewable energy usages and fulfil the requirement of operating a generating station. Therefore, power generating cost and carbon emission cost are combined in this objective function. The cost function can be defined as follows:

$$
F_{C E}=F_{C}+C_{t} \cdot F_{E}
$$

where $C_{t}$ represents the carbon tax and it is considered $20(\$ / \mathrm{h})[38]$.

\subsection{Constraints}

While minimizing the objective function of the OPF problem, it is essential to fulfill the equality and inequality constraints to reliably and securely operate a power system. The constraints of the OPF problem are described in the following subsection.

\subsubsection{Equality constraints}

The total power generation should be equal to the total power demand of the network considering power losses in the transmission, otherwise, an unbalanced situation will create, leading to unstable in the power system. The equality constraints embody the non-linear power flow equations as follows: 
(a) Real power constraints

$$
P_{G_{i}}-P_{D_{i}}-V_{i} \sum_{j=i}^{N B} V_{j}\left[G_{i j} \cos \left(\theta_{i j}\right)+B_{i j} \sin \left(\theta_{i j}\right)\right]=0
$$

(b) Reactive power constraints

$$
Q_{G_{i}}-Q_{D_{i}}-V_{i} \sum_{j=i}^{N B} V_{j}\left[G_{i j} \sin \left(\theta_{i j}\right)-B_{i j} \cos \left(\theta_{i j}\right)\right]=0
$$

where $\theta_{i j}=\theta_{i}-\theta_{j}, P_{D}$ is the active power demand, $Q_{D}$ is the reactive power demand, $N B$ is the number of buses, $G_{i j}$ and $B_{i j}$ are the conductance and susceptance between buses $i$ and $j$, respectively.

\subsubsection{Inequality constraints}

There are a number of inequality constraints that reflect the physical limits of the power system and system operation. These are expressed mathematically below.

(a) Generator constraints

All the voltage, active power and reactive power of generators should be restricted by their upper and lower limits as follows:

$$
\begin{aligned}
V_{G_{i}}^{\min } \leq V_{G_{i}} \leq V_{G_{i}}^{\max }, & i=1,2, \ldots, N G \\
P_{G_{i}}^{\min } \leq P_{G_{i}} \leq P_{G_{i}}^{\max }, & i=1,2, \ldots, N G \\
Q_{G_{i}}^{\min } \leq Q_{G_{i}} \leq Q_{G_{i}}^{\max }, & i=1,2, \ldots, N G
\end{aligned}
$$

where $V_{G_{i}}^{\min }$ and $V_{G_{i}}^{\max }$ are the minimum and maximum generation voltage of the $i$ th generating units, respectively; $P_{G_{i}}^{\min }$ and $P_{G_{i}}^{\max }$ are the minimum and maximum active power output of the $i$ th generating units, respectively; and $Q_{G_{i}}^{\min }$ and $Q_{G_{i}}^{\max }$ are the minimum and maximum reactive power output of the $i$ th generating unit, respectively. It should be noted that $i=3$ and $i=5$ are the limits of wind power generators and $i=6$ solar generators. As the OPF problem is solved in a real-time power dispatch mode, these limits become the same with the thermal generators. In practice, these limits vary in every hour as power generation from renewable energy sources are intermittent and these can be calculated using probability distribution function. Although rooftop solar panels are not generally prepared for reactive power supply, solar and wind power generators in utility-scale have the capability to supply reactive powers into the grid $[41,42]$.

(b) Security constraints

The security constraints include voltage magnitudes at load buses and transmission line flow. The operational limits of these components must be fulfilled by their upper and lower limits as follows:

$$
\begin{array}{r}
V_{L_{i}}^{\min } \leq V_{L_{i}} \leq V_{L_{i}}^{\max }, \quad i=1,2, \ldots, N L \\
S_{l_{i}} \leq S_{l_{i}}^{\max }, \quad i=1,2, \ldots, n l
\end{array}
$$

where $V_{L_{i}}^{\min }$ and $V_{L_{i}}^{\max }$ define the minimum and maximum load voltages of the $i$ th unit; $S_{l_{i}}$ is the apparent power flow limit of the $i$ th branch.

\section{Proposed Algorithm}

As previously stated, the relative performance of DE algorithm may vary during the optimization stages; in other words, the performance of one DE operator may be good at the early phases of the optimization process but worse at the later phases, or vice versa [32, 30, 29]. Also, according to the free-lunch theorem, an operator may perform well for certain types of a problem but its performance may be bad for another. Moreover, balancing the diversification 
and intensification during the optimization process is crucial in designing optimization algorithms [43]. As a result, the use of multi-operator DE is required with placing more weight to the better-performing one at each evolutionary stage and managing the balance between the exploration and exploitation. In this paper, we propose a multi-operator DE (MODE) optimization algorithm to solve the OPF problem, while its main steps are presented in Algorithm 1 and shown in Figure 6.

The proposed MODE-OPF starts with a random initial population of size NP solutions (i.e., $x$ and $u$ are generated). Then, the objective function value $(F(x, u))$ and total constrained violation $(\psi(x, u))$ for each solution is computed. Initially, each DE operator is used to guide the same number of solutions $P S_{o p}$ towards the optimal solution, i.e., new individuals are produced by its assigned DE mutation strategy. The objective function value and constraint violations for the newly generated populations are computed. Then, a pairwise comparison between each individual in the parent population and its corresponding one in the offspring population is carried out (discussed in Subsection 3.4), with the winner enters the next generation. Subsequently, at the end of each iteration, the improvement index is calculated (discussed in Subsection 3.2). Based on the improvement index, the number of individuals that evolved by every DE mutation strategy is updated. A minimum number of individuals evolved by each DE mutation strategy was set. Indeed, MODE-OPF uses a linear population size reduction approach at the end of each iteration by removing the worst individual [44, 43], as in Equation 27. This is done to preserve the diversity at the early generations and boosting the convergence in the later ones.

$$
N P_{t+1}=\operatorname{round}\left[\left(\frac{N P_{\text {min }}-N P_{\text {init }}}{M A X_{F E S}}\right) \times F E S+N P_{\text {init }}\right]
$$

where $N P_{\text {init }}$ is the initial population size, $N P_{\min }$ the minimum number of solutions the solver can use, $F E S$ the current number of function evaluated (FES) and MAX $X_{F E S}$ the largest number of FES.

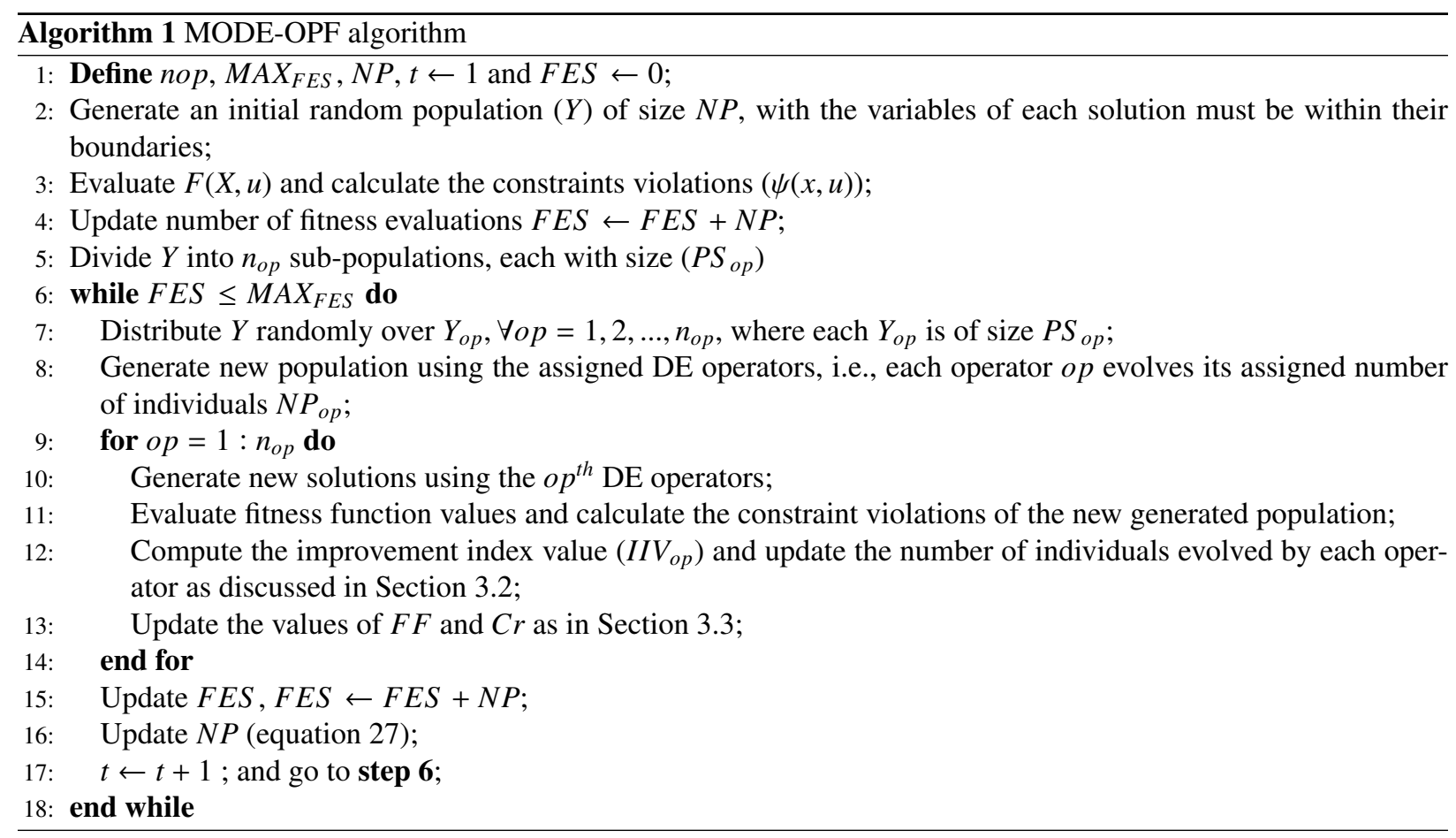

The following subsections provide detail information about the components of the proposed MODE-OPF.

\subsection{DE mutation strategies}

MODE-OPF uses the following DE mutation strategies to evolve the whole population as they perform well in solving unconstrained and constrained optimization problems [33, 45, 31]. 


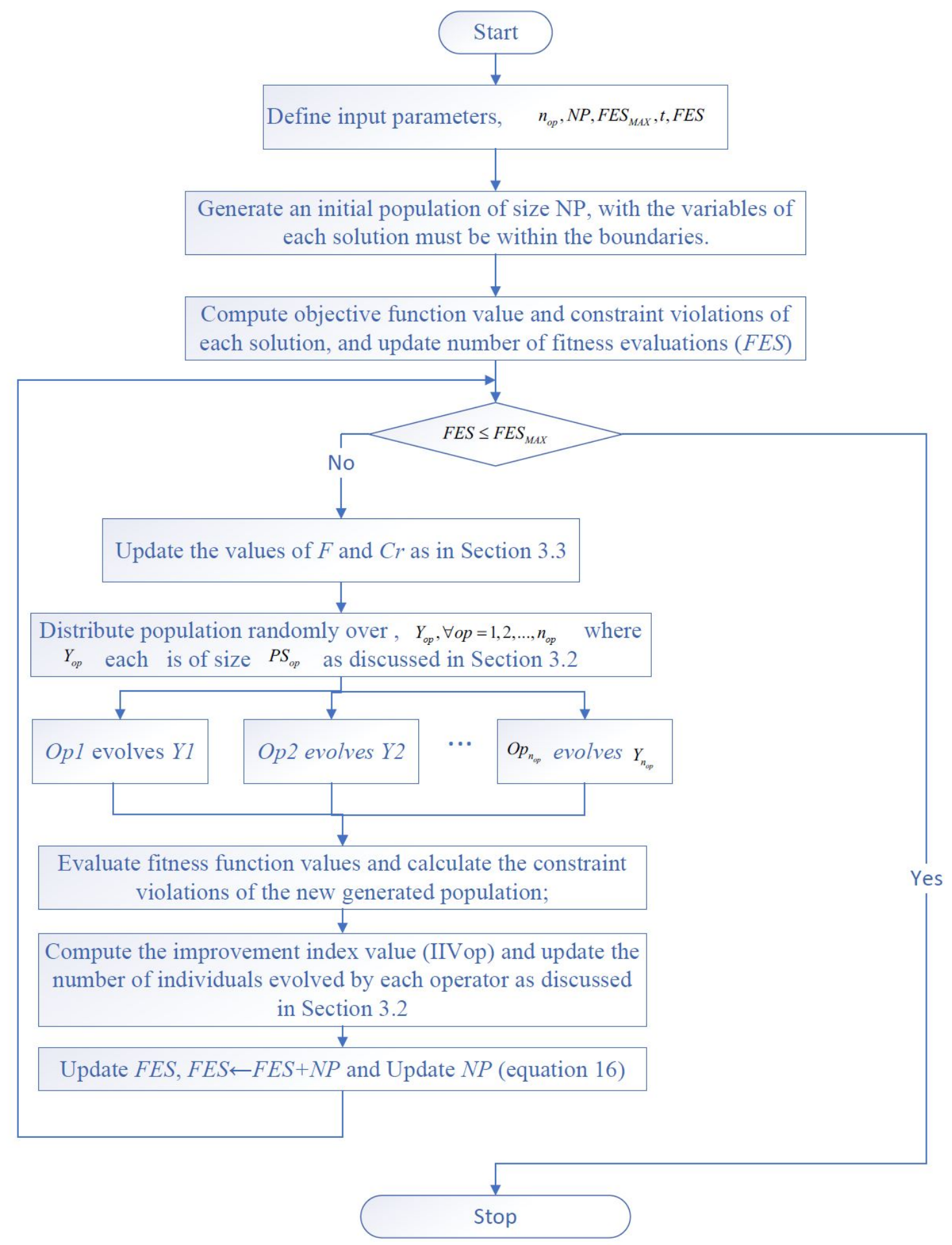

Figure 6: Flowchart of the proposed MODE-OPF 
- $\mathrm{DE} /$ rand-to- $\phi$ best/1/bin with archive

$$
u_{i, j}=\left\{\begin{array}{r}
x_{r 1, j}+F F_{i \cdot}\left(x_{\phi, j}-x_{r 1, j}+x_{r 2, j}-x_{r 3, j}\right) \\
\text { if }\left(\text { rand } \leq C r_{i} \text { or } j=j_{\text {rand }}\right) \\
x_{i, j} \text { otherwise }
\end{array}\right.
$$

- $\mathrm{DE} /$ current-to- $\phi$ best/1/bin with archive

$$
u_{i, j}=\left\{\begin{array}{rr}
x_{i, j}+F F_{i \cdot}\left(x_{\phi, j}-x_{i, j}+x_{r 1, j}-x_{r 3, j}\right) \\
\text { if }\left(\text { rand } \leq C r_{i} \text { or } j=j_{\text {rand }}\right) \\
x_{i, j} \quad \text { otherwise }
\end{array}\right.
$$

- DE weighted-rand-to- $\phi$ best

$$
u_{i, j}=\left\{\begin{array}{rr}
F F \times x_{r_{1}, j}+\left(x_{\phi, j}-x_{r_{2}, j}\right) & \\
& \text { if }\left(\text { rand } \leq C r_{i} \text { or } j=j_{\text {rand }}\right) \\
x_{i, j} & \text { otherwise }
\end{array}\right.
$$

where $r_{1} \neq r_{2} \neq r_{3} \neq i$ are integers numbers randomly generated, $\vec{x}_{r 1}$ and $\vec{x}_{r 2}$ randomly picked up from the entire population, with $x_{\phi, j}$ drawn up from the best $10 \%$ of individuals in the entire population and $\vec{x}_{r 3}$ from the union of the whole population and archive. An archive to maintain the population's diversity, is used. New candidates that are worse than their parent ones kept in the archive [46]. To make space for the newly generated individuals, once the archive size is greater than its default size, the worst individuals are removed from it.

Note that the proposed framework is flexible to adopt more operators.

\subsection{Updating number of solutions evolved by operator op $\left(N P_{o p}\right)$}

As we previously mentioned, the solutions' quality and sub-population's diversity are used to update the number of individuals evolved by each DE mutation operator. Consequently, this method is referred as adaptive method (AM). For the solutions' quality, the best individual at the end of each iteration $(t)$ that is found by each DE operator is used to calculate the normalized quality $\left(Q_{u a l}\right)$ for operator $o p$ as

$$
\text { Qual }_{o p}=\frac{F_{t, o p}^{\text {best }}}{\sum_{o p=1}^{n_{o p}} F_{t, o p}^{\text {best }}}, \forall o p=1,2, \ldots, n_{o p}
$$

where $F_{t, o p}^{\text {best }}$ is the best objective function value obtained by DE operator $o p$ at generation $t$. Note, as we deal with constrained optimization problem, $F_{t, o p}^{b e s t}$ is determined based on both the fitness function values and the total constraint violations. This is done by sorting the solutions based on both fitness function values and total constraint violations [47].

Similarly, for the diversity obtained from each DE search operator $\left(D i v_{o p}\right)$ which is the mean deviation of each solution obtained from $o p$ of the best solution computed as

$$
D i v_{o p}=\frac{1}{N P_{o p}}\left(\sum_{i=1}^{N P_{o p}} \operatorname{dis}\left(\vec{U}_{o p, i}-\vec{U}_{o p}^{b e s t}\right)\right), \forall o p=1,2, \ldots, n_{o p}
$$

where $\operatorname{dis}\left(\vec{U}_{o p, i}-\vec{U}_{o p}^{b e s t}\right)$ is the Euclidean distance between the $i^{\text {th }}$ solution and best one obtained by each operator $o p$. Also the diversity index is calculated by:

$$
D I_{o p}=\frac{D i v_{o p}}{\sum_{o p=1}^{n_{o p}} D i v_{o p}}, \forall o p=1,2, \ldots, n_{o p}
$$


Based on the above equations, the improvement index value $\left(I I V_{o p}\right)$ is calculated as:

$$
I I V_{o p}=(1-c) \times\left(1-Q_{u a l}\right)+c \times D I_{o p}, \forall o p=1,2, \ldots, n_{o p}
$$

Note: to satisfy the aim of maximizing the $I I V_{o p}$, we subtracted $Q u a l_{o p}$ from 1 . In this paper, in order to put more weight on the DE operator that generate more diverse population at the early stages of the optimization process, while focus on the one that has a faster convergence at the later stages, the parameter $c$ is defined in Equation 35 .

$$
c=1-\frac{F E S}{M A X_{F E S}}
$$

Finally, the number of individuals that each DE mutation strategy $\left(N P_{o p}\right)$ evolves is computed by

$$
N P_{o p}=\max \left(0.1, \min \left(0.9, \frac{I I V_{o p}}{\sum_{o p=1}^{n_{o p}} I I V_{o p}}\right)\right) \times N P,
$$

Note: $\sum_{o p=1}^{n_{o p}} N P_{o p}=N P$, i.e., the sum of all individuals evolved by each DE mutation strategy must be equal to the whole population size. Also, as one of the DE operators may differently behave during the different stages of the evolutionary process, i.e., it may perform good at the early stages and behave differently at the the latter stage [29]. Thus, we use a minimum value (0.1) to give a chance of the low-performing operators to be applied.

\subsection{Managing $\mathrm{FF}$ and $\mathrm{Cr}$}

The DE's performance is highly dependent upon its search operators (mutation and crossover) and its control parameters $(N P, F F$ and $C r)$. However, choosing their values is challenging. Thus, in this paper, a self-adaptive method is used to update the values of $F F$ and $\operatorname{Cr}[33,31]$. To do this, the following steps are performed:

- A recording memory that has $L$ elements for $\mu F F$ and $\mu C r$ is initialized, with the values of all elements are initially set to 0.5 .

- Each individual $\vec{U}_{i}$ is associated with its own $F F_{i}$ and $C r_{i}$ values, such that:

$$
\begin{gathered}
F F_{i}=\operatorname{randci}\left(\mu F F_{r_{i}}, 0.1\right) \\
C r_{i}=\operatorname{randni}\left(\mu C r_{r_{i}}, 0.1\right)
\end{gathered}
$$

where $r_{i}$ is picked randomly from $[1, L]$, randci and randni are values randomly generated by using Cauchy and Normal distributions with variance 0.1 , mean $\mu F F$ and $\mu C r$, respectively [44].

- At the end of each iteration $t, F F_{i}$ and $C r_{i}$ used by the successful individuals are saved in $S F F$ and $S C r$, and then the elements of the recording memory are updated as follows:

$$
\begin{gathered}
\mu F F_{l}=\operatorname{mean}_{W L}(S F F) \text { if } S F F \neq \text { null } \\
\mu C r_{l}=\operatorname{mean}_{W A}(S C r) \text { if } S C r \neq \text { null }
\end{gathered}
$$

where $1 \leq l \leq L$ is the the location in the recording memory to be updated, with its initial value is 1 , and then increased whenever a new element is stored into the recording memory, and if its size is larger than $\mathrm{L}$, it is reset to 1 . The Lehemer mean $\left(\operatorname{mean}_{W L}(S F F)\right)$ and the weighted mean $\left(\operatorname{mean}_{W A}(S C r)\right)$ are calculated as follows: 


$$
\begin{gathered}
\operatorname{mean}_{W L}(S F F)=\frac{\sum_{\zeta=1}^{|S F F|} \omega_{\zeta} S F F_{\zeta}^{2}}{\sum_{\zeta=1}^{|S F F|} \omega_{\zeta} S F F_{\zeta}} \\
\operatorname{mean}_{W A}(S C r)=\sum_{\zeta=1}^{|S C r|} \omega_{\zeta} S C r_{\zeta}
\end{gathered}
$$

where $|\mathrm{SFF}|$ and $|\mathrm{SCr}|$ are the number of successful $F F$ and $C r$ saved in $S F F$ and $S C r$ with $|\mathrm{SCr}|=|\mathrm{SF}|$ and $\omega_{\zeta}$ is the weight calculated by:

$$
\omega_{\zeta}=\frac{\gamma_{\zeta}}{\sum_{\zeta=1}^{\left|S_{C r}\right|} \gamma}
$$

The values of $\gamma_{\zeta}$ are calculated based on the following scenarios:

i Feasible to feasible: the violation of the best solution equal zero at both generations $t-1$ and $t$;

ii Infeasible to feasible: at iteration $t-1$, the best candidate solution in the population is infeasible and then it becomes feasible at generation $t$; and

iii Infeasible to infeasible: the best candidate solution in the population is infeasible in generations $t-1$ and $t$.

First, for each successful solution $\left(h \in 1,2, \ldots,\left|S_{C r}\right|\right)$ which falls in scenario (i), its $\gamma_{h}$ is calculated as [33, 31]:

$$
\gamma_{h}=\beta_{h=} \max \left(0, \frac{\psi_{h, t-1}-\psi_{h, t}}{\psi_{h, t-1}}\right)+\max \left(0, \frac{F_{h, t-1}-F_{h, t}}{F_{h, t-1}}\right)
$$

Then, for each successful solutions which exists in scenarios 2 or 3 , its $\gamma_{h}$ is computed as:

$$
\gamma_{h}=\max \left(0, \beta_{h}\right)+\frac{\psi_{h, t-1}-\psi_{h, t}}{\psi_{h, t-1}}+\max \left(0, \frac{F_{h, t-1}-F_{h, t}}{F_{h, t-1}}\right)
$$

\subsection{Constraints handling}

It is well known that the greater the number of constraints (equality and inequality) exist in any constrained optimization problem, the more complex and complicated will be that problem. One way of dealing with this issue is to use a subset of the constraints to calculate the total constraints violations, and gradually increase this number until the total number of constraints are handled. In this paper, we used the constrained handling technique proposed in [48]. The method starts by sorting all the constraints based on their sum of the constraint violations of all the solutions in the initial population. It begins by ordering the constraints according to the sum of the constraint violations of all the individuals in the initial population from the most violated to least violated constraint or vice versa. Then, instead of using all constraints, the proposed MODE-OPF starts with a small number of them $\left(\right.$ Con $\left._{n}\right)$ (the ones with the largest total violation) for a predetermined number of iterations $(W)$, then a new subset is appended to the current set, and the method tries to attain the feasible region of both the new and previous subsets of constraints. This process lasts until all the constraints are handled and the final feasible region is reached.

To select between any solution $B_{\text {new }}$ and its parent $B_{\text {old }}$, the method developed by Deb [47] is adopted and it has three scenarios:

1. If both $B_{\text {old }}$ and $B_{\text {new }}$ are feasible and $f\left(B_{\text {new }}<f\left(B_{\text {old }}\right)\right.$, the $B_{\text {new }}$ is selected;

2. If both $B_{\text {old }}$ and $B_{\text {new }}$ are infeasible and $\psi\left(B_{\text {new }}\right)<\psi\left(B_{\text {old }}\right)$, then $B_{\text {new }}$ is chosen, where $\psi$ is computed using Equation 46; and

3. If $B_{\text {new }}$ is feasible and $B_{\text {old }}$ is infeasible, then $B_{\text {new }}$ is preferred. 


$$
\psi\left(\vec{x}_{i}\right)=\sum_{k=1}^{K} \max \left(0, g_{k}\left(\vec{x}_{i}\right)\right)+\sum_{e=1}^{E} \max \left(0,\left|h_{e}\left(\vec{x}_{i}\right)\right|-\delta_{e}\right)
$$

where $g_{k}\left(\vec{x}_{i}\right)$ and $h_{e}\left(\vec{x}_{i}\right)$ are the $k^{\text {th }}$ inequality and $e^{\text {th }}$ equality constraints, respectively. For each equality constraint $h_{e}, \delta_{e}$ was set to a value of 0.0001 [31].

\section{Experimental results and Analysis}

In this paper, to test the performance of the proposed MODE-OPF, an updated IEEE 30-bus system has been considered in line to the recent papers published in $[12,39,49,38]$ as depicted in Figure 7 . In this modified system, the conventional thermal generators are substituted for wind turbines on bus 5, 11 and the solar generators on bus 13 . The intermittent power generation cost for the wind power generators are determined by the combination of scheduling cost, overestimated cost and underestimated cost, represented in Eqs. (3), (4), and (5), respectively. Similarly, the cost of intermittent solar power is estimated by adding the cost involve in scheduling, overestimating and underestimating as represented in Eqs. (11), (12) and (13), respectively. Bus 1 of the network is used as the swing or slack bus that is responsible for maintaining balanced active and reactive power flow in the network while fulfilling equality constraints. As swing bus is considered as a reference bus, its voltage magnitude and angle are 1 p.u. and 0 degree, respectively. All other bus voltages and angles are computed with respect to the swing bus using load flow study. The power flow problem is solved using the Newton-Raphson iterative method under the MATPOWER tool [50]. Table 1 shows the parameters of renewable energy sources and the coefficients of thermal generators are shown in Table 2. Table 4 presents the lower and upper bounds of the control and state variables [38].

We coded and implemented all the algorithms in MATLAB R2018b and ran them on a PC with 16GB RAM, core I7 processor with a $3.4 \mathrm{GHz}$ and Windows10. Note that all the rival algorithms are executed 30 times, with the best, median, average, worst and standard deviation of results reported. Results are recorded with a stopping condition equal to 20000 and 100000 fitness evaluations (i.e., FES ${ }_{M A X}$ equal to 20000 or 100000) for IEEE 30 and IEEE 118 bus systems, respectively. The parameter settings of the competitive algorithms are taken from their original sources. For a fair comparison, the population sizes of all the compared algorithms including the proposed one is set to 50 . To test and judge the performance of the proposed MODE-OPF, we carried out a comparison with the following algorithms:

1. A genetic algorithm with multi-parent crossover (GA-MPC) [51];

2. An enhanced JADE algorithm with self-adaptive penalty constraint handling method (EJADE-SP) [12];

3. A DE with an ensemble of constraints handling technique (ECHT-DE) [52];

4. A unified DE for constrained optimization (UDE) [53];

5. An LSHADE with competing strategies for constrained optimization (LSHADE44) [54];

6. A composite DE algorithm for solving constrained optimization problems (COPs) $\left(\mathrm{C}^{2} \mathrm{oDE}\right)[55]$;

7. A feasibility rule with the incorporation of objective function information (FROFI). [56]

We conducted two non-parametric tests (Wilcoxon signed-ranked test with a 0.05 significance and Friedman's ranking test) [57] to statistically compare the rival algorithms. Two algorithms $A l g_{1}$ and $A \lg _{2}$ are compared using the Wilcoxon signed-rank test, with one of the following signs used.

- The "+ " sign that means $A \lg _{1}$ algorithm is significantly superior to $\mathrm{Alg}_{2}$;

- The "- " sign that means $A \lg _{1}$ is significantly inferior to $A \lg _{2}$; and

- The " $\approx "$ sign that means there is no significant difference between $A l g_{1}$ and $A \lg _{2}$. 


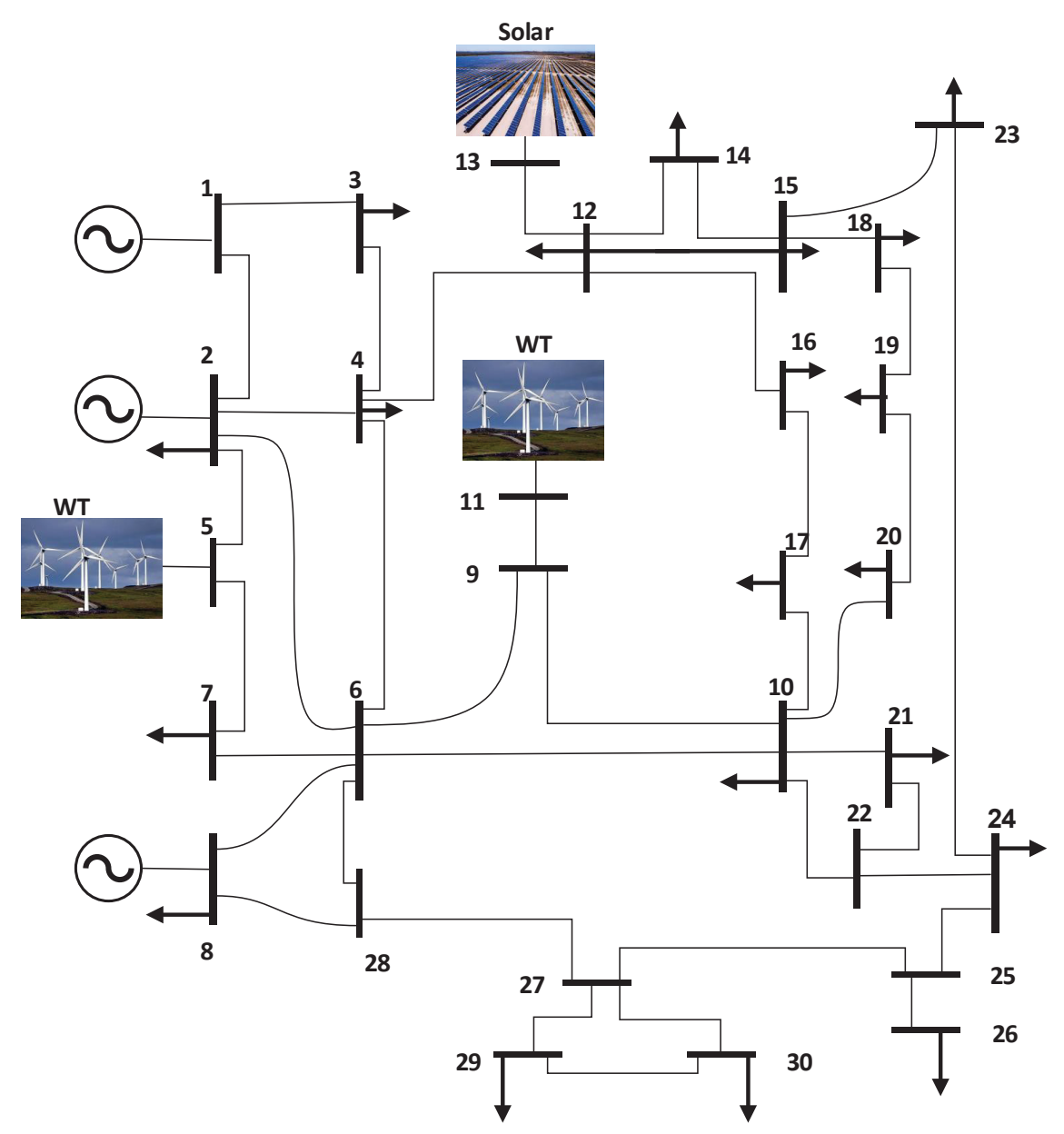

Figure 7: Diagram of modified IEEE 30-bus test system

Table 1: Parameters of solar and wind generation [58]

\begin{tabular}{cccc|c}
\hline \# of wind-farms & \# of turbine & Ratings & Total rated power & Solar power rating \\
\hline 1 (bus 5) & 25 & $3 \mathrm{MW}$ & $75 \mathrm{MW}$ & $50 \mathrm{MW}$ (bus 13) \\
2 (bus 11) & 20 & $3 \mathrm{MW}$ & $60 \mathrm{MW}$ & \\
\hline
\end{tabular}

Table 2: Coefficient of thermal power generator

\begin{tabular}{|c|c|c|c|c|c|c|c|c|c|c|}
\hline Generators & $\mathbf{a}(\$ / \mathbf{h})$ & $\mathbf{b}(\mathbf{\$} / \mathbf{M W h})$ & c (\$ / $\left./ \mathbf{M W}^{2} \mathbf{h}\right)$ & $\mathrm{d}(\mathbf{\$} / \mathbf{h})$ & e (rad/MW) & $\alpha(\mathbf{t} / \mathbf{h})$ & $\beta$ (t//p.u.MWh) & $\gamma\left(\mathbf{t} /\right.$ p.u.MW $\left.{ }^{2} \mathbf{h}\right)$ & $\omega(\mathbf{t} / \mathbf{h})$ & $\mu\left(\right.$ p.u.MW $\left.{ }^{-} 1\right)$ \\
\hline$P_{G 1}$ (bus-1) & 30 & 2 & 0.00375 & 18 & 0.037 & 0.04091 & -0.05554 & 0.0649 & 0.0002 & 6.667 \\
\hline$P_{G 2}$ (bus- 2 ) & 25 & 1.75 & 0.0175 & 16 & 0.038 & 0.02543 & -0.06047 & 0.05638 & 0.0005 & 3.333 \\
\hline$P_{G 4}$ (bus-8) & 20 & 3.25 & 0.00834 & 12 & 0.045 & 0.05326 & -0.0355 & 0.0338 & 0.002 & 2 \\
\hline
\end{tabular}


Table 3: An overview of IEEE 30-bus system

\begin{tabular}{ccc}
\hline Items & Quantity & Details \\
Buses & 30 & {$[59]$} \\
Branches & 41 & {$[59]$} \\
Wind generators & 3 & Buses: 1 (swing), 2 and 8 \\
Solar generators & 2 & Buses: 5 and 11 \\
Control variables & 1 & Bus: 13 \\
& 11 & Active power of five generators except swing bus, \\
Connected load & - & and bus voltages of all generators (six) \\
Load bus voltage allowed & 24 & 283.4 MW, 126.2 MVAr \\
\hline
\end{tabular}

\begin{tabular}{cccccc} 
Table 4: The lower and upper bounds of state and control variables of 30-bus system \\
\hline Control variables & LB & UB & State variables & LB & UB \\
\hline$P_{G_{2}}$ & 20 & 80 & $P_{G_{1}}$ & 30 & 140 \\
$P_{G_{3}}$ & 0 & 75 & $Q_{G_{1}}$ & -20 & 150 \\
$P_{G_{4}}$ & 10 & 35 & $Q_{G_{2}}$ & -20 & 60 \\
$P_{G_{5}}$ & 0 & 60 & $Q_{G_{3}}$ & -15 & 40 \\
$P_{G_{6}}$ & 0 & 50 & $Q_{G_{4}}$ & -30 & 35 \\
$v_{1}$ & 0.95 & 1.10 & $Q_{G_{5}}$ & -25 & 30 \\
$v_{2}$ & 0.95 & 1.10 & $Q_{G_{6}}$ & -20 & 25 \\
$v_{5}$ & 0.95 & 1.10 & & & \\
$v_{8}$ & 0.95 & 1.10 & & & \\
$v_{11}$ & 0.95 & 1.10 & & & \\
$v_{13}$ & 0.95 & 1.10 & & & \\
\hline
\end{tabular}

\subsection{Effect of number of used DE operators}

The proposed MODE-OPF uses more than one DE mutation strategies in a single algorithmic system. Thus, in order to design an effective multi-operator algorithm, it is mandatory to know which combination of single operators is better to be used in the framework [32,45]. To do this, in this section a comparison is conducted between seven variants of the proposed algorithm. These variants share the same structure and components of the proposed algorithm and they are different only in the number and operators they used. These variants are as follows:

1. Var1: DE/rand-to- $\phi$ best/1/bin with archive is used to evolve the entire population.

2. Var2: DE/current-to- $\phi$ best $/ 1 /$ bin with archive is used to evolve the entire population.

3. Var3: DE/weighted-rand-to- $\phi$ best/1/bin is used to evolve the entire population.

4. Var4: Both DE/rand-to- $\phi$ best $/ 1 /$ bin with archive and DE/current-to- $\phi$ best $/ 1 /$ bin with archive are used to generate new solutions.

5. Var5: Both DE/rand-to- $\phi$ best/1/bin with archive and DE/weighted-rand-to- $\phi$ best/1/bin with archive are used to generate new solutions.

6. Var6: Both DE/current-to- $\phi$ best/1/bin and DE/weighted-rand-to- $\phi$ best/1/bin with archive are used to generate new solutions.

7. Var7: All three operators (DE/rand-to- $\phi$ best/1/bin with archive, $D E /$ current-to- $\phi$ best/1/bin with archive and $\mathrm{DE} /$ weighted-rand-to- $\phi$ best $/ 1 /$ bin) are used to evolve the entire population.

All seven variants are tested and compared against each other by solving the OPF optimization problem with the objective function to minimize the cost of generation (case 1). The parameters values used in these variants and the proposed MODE-OPF algorithm through the paper were set as follows: the maximum number of solutions $N P_{\text {init }}$ equal to 50, while the minimum number of individuals $N P_{\min }$ equal to 30 . The value of $\phi$ was set to 0.11 for the first two DE operators and 0.5 for the third, the archive rate $(A)$ to 1.4 , and the memory size $(L)$ to 5 [29]. The window size, $W$, was set at a value of 50 [48]. 
Table 5 presents the summary of the results obtained from the seven variants. From Table 5, it is clear that Var4 obtains the best median, best average and best maximum values of the generation cost, while Var1 archives the best minimum value in comparison to others with a very different value in cost from Var4 (0.0000014).

Table 5: Comparative summary of seven variants for the algorithm proposed on Case 1

\begin{tabular}{cccccc}
\hline Algorithms & Best & Median & Mean & Worst & Std. \\
\hline Var1 & $\mathbf{7 8 2 . 3 5 9 2 7 5 0}$ & 782.3755527 & 782.3683531 & 782.3759836 & 0.0080680 \\
Var2 & 782.3592753 & 782.3755724 & 782.3686045 & 782.3756127 & 0.0081140 \\
Var3 & 782.3592852 & 782.3755351 & 782.3702135 & 782.3760232 & 0.0071990 \\
Var4 & 782.3592758 & $\mathbf{7 8 2 . 3 5 9 3 4 6 6}$ & $\mathbf{7 8 2 . 3 6 5 3 4 6 2}$ & $\mathbf{7 8 2 . 3 7 5 5 8 0 6}$ & 0.0082426 \\
Var5 & 782.3592910 & 782.3755744 & 782.3708036 & 782.3757930 & $\mathbf{0 . 0 0 7 2 1 1 9}$ \\
Var6 & 782.3592818 & 782.3693282 & 782.3676928 & 782.3756323 & 0.0080616 \\
Var7 & 782.3592764 & 782.3594074 & 783.668761 & 782.3762862 & 0.0079734 \\
\hline
\end{tabular}

To further statistically compare all seven variants, we carried out a Wilcoxon signed-rank test [36]. The summary of the experimental results obtained from the Wilcoxon signed-rank test is presented in Table 6. It can be concluded from Table 6 that Var4 is statistically better than Var3, Var5 and Var6, while there is no significant difference statistically between Var4 and other variants, although there is a bias in the results towards Var4.

\begin{tabular}{|c|c|c|c|c|}
\hline Algorithms & $R^{+}$ & $R^{-}$ & Exact P-value & Dec. \\
\hline Var4 vs. Var1 & 297.0 & 168.0 & 0.181242 & $\approx$ \\
\hline Var4 vs. Var 2 & 302.0 & 163.0 & 0.149929 & $\approx$ \\
\hline Var4 vs. Var3 & 342.0 & 123.0 & 0.023665 & + \\
\hline Var4 vs. Var5 & 360.0 & 105.0 & 0.00847 & + \\
\hline Var4 vs. Var6 & 363.5 & 101.5 & 0.006694 & + \\
\hline Var4 vs. Var7 & 237.0 & 228.0 & 0.917878 & $\approx$ \\
\hline
\end{tabular}

As a further analysis, Friedman's rank test has been conducted among the seven variants, while the results are depicted in Figure 8. It is seen in Figure 8 that Var4 has the first rank, followed by Var7, then Var1. From the above analysis, it can be concluded that using two DE mutation strategies (Var4: DE/rand-to- $\phi$ best/1/bin with archive and DE/current-to- $\phi$ best/1/bin with archive) is better than all other variants based on both Wilcoxon signed-rank and Friedman rank tests.

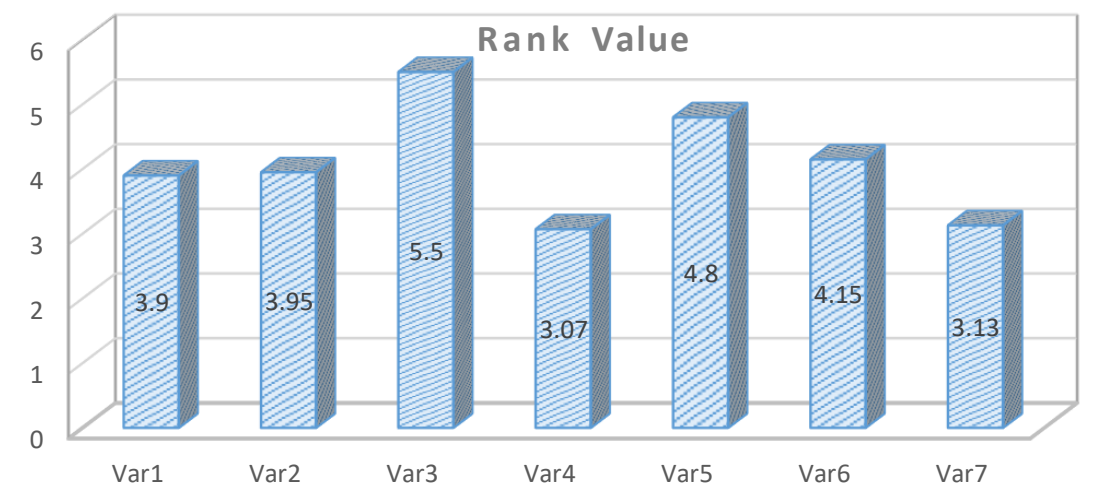

Figure 8: Average ranking achieved by Friedman's ranking test to compare the seven variants on Case 1

\subsection{Comparison with the state-of-the-art algorithms for the IEEE 30-bus system}

As previously stated, the performance of the proposed MODE-OPF is tested by optimizing five different objective functions of the power flow optimization problems. These five cases are discussed in details by providing their 
mathematical formulations at Subsection 2.3. In the following subsections, the detailed results obtained from the proposed algorithm along with a comparison with the state-of-the-art algorithms are discussed.

\subsubsection{Results of case 1}

The objective function at this case is to find the optimal settings of generators for which power generation cost can be minimized (i.e., the operating cost of power generation can be saved). The lower value in solving the objective function represents the minimum operating cost of generating units per hour to run the power system efficiently and effectively. Table 7 presents the summary of comparison for generation cost obtained from the proposed MODE-OPF and the rival algorithms based on minimum, maximum, median, average and standard deviation of the generation cost. Considering the summary of the fuel cost obtained in Table 7, MODE-OPF is better than EJADE-SP, C ${ }^{2}$ oDE, LSHADE44, UDE, ECHT-DE and FROFI in regards to minimum, median, average and maximum cost generation. Therefore, it can be concluded that the proposed solver has the capability to reduce the total fuel cost by $0.0048 \%$, $2.11 \%, 1.64 \%, 24.08 \%, 2.40 \%$ and $4.39 \%$ as compared to EJADE-SP, C ${ }^{2}$ oDE, LSHADE44, ECHT-DE, FRORI and GA-MPC, respectively. It can be also observed from Figure 15 that the fuel cost is less as compared to the other cases while other parameters like power losses, voltage deviation and emission are higher as shown in Table 18.

\begin{tabular}{cccccc}
\multicolumn{7}{c}{ Table 7: Case 1: Comparative summary of the algorithms } \\
\hline Algorithms & Best & Median & Mean & Worst & Std. \\
\hline GA-MPC & 782.4032000 & 784.8747000 & 787.9558267 & 835.9240000 & 11.1466094 \\
EJADE-SP & 782.3593253 & 782.4638016 & 782.3716416 & 782.3751388 & 0.0190688 \\
C $^{2}$ oDE & 782.3804017 & 782.4352287 & 782.3987796 & 782.3969633 & 0.0136890 \\
LSHADE44 & 782.3757000 & 782.7229000 & 782.4513700 & 782.3790500 & 0.1097607 \\
UDE & 782.3592753 & 782.7213908 & 782.4037119 & 782.3755732 & 0.0897619 \\
ECHT-DE & 782.6001000 & 782.8470000 & 782.6924167 & 782.6882000 & 0.0568796 \\
FROFI & 782.3833028 & 782.4983369 & 782.4169422 & 782.4159308 & 0.0239930 \\
MODE-OPF & $\mathbf{7 8 2 . 3 5 9 2 7 6 4}$ & $\mathbf{7 8 2 . 3 7 6 2 8 6 2}$ & $\mathbf{7 8 2 . 3 6 5 3 4 6 2}$ & $\mathbf{7 8 2 . 3 5 9 4 0 7 4}$ & $\mathbf{0 . 0 0 7 9 7 3 4}$ \\
\hline
\end{tabular}

Referring to the summary of the results obtained from the Wilcoxon signed-rank test as shown in Table 8 , the proposed MODE-OPF is significantly better than all the competiting algorithms (EJADE-SP, C ${ }^{2}$ oDE, LSHADE44, UDE, ECHT-DE, FROFI and GA-MPC) with the p-value less than 0.05 in all cases. Meanwhile, Figure 9 demonstrates the Friedman rank test and it is observed that the proposed MODE-OPF was ranked first, followed by EJADE-SP, and then UDE.

Table 8: Case 1: Results obtained by the Wilcoxon test for the algorithm proposed

\begin{tabular}{ccccc}
\hline Algorithms & $R^{+}$ & $R^{-}$ & Exact P-value & Dec. \\
\hline MODE-OPF vs. EJADE-SP & 339.0 & 126.0 & 0.02774 & + \\
MODE-OPF vs. C ${ }^{2}$ oDE & 465.0 & 0.0 & $1.8626 \mathrm{E}-9$ & + \\
MODE-OPF vs. LSHADE44 & 460.0 & 5.0 & $1.8626 \mathrm{E}-8$ & + \\
MODE-OPF vs. UDE & 351.0 & 114.0 & 0.013664 & + \\
MODE-OPF vs. ECHT-DE & 465.0 & 0.0 & $1.8626 \mathrm{E}-9$ & + \\
MODE-OPF vs. FROFI & 465.0 & 0.0 & $1.8626 \mathrm{E}-9$ & + \\
MODE-OPF vs. GA-MPC & 465.0 & 0.0 & $1.8626 \mathrm{E}-9$ & + \\
\hline
\end{tabular}

\subsubsection{Results of case 2}

The net power obtained by the distribution grid is the difference between the overall power output and transmission power losses. Thus, the minimization of power losses is the best way to increase the performance of the distribution system. As a result, the minimization of power losses is a vital problem in the power systems. In case 2, the proposed MODE-OPF is used to minimize the power loss, which is considered as the fitness function for this case. The summary of the results for minimum, median, average and maximum obtained from the proposed MODE-OPF, EJADE-SP, $\mathrm{C}^{2} \mathrm{ODE}$, LSHADE44, UDE, ECHT-DE and FROFI is presented in Table 9. Considering the summary of the statistical 


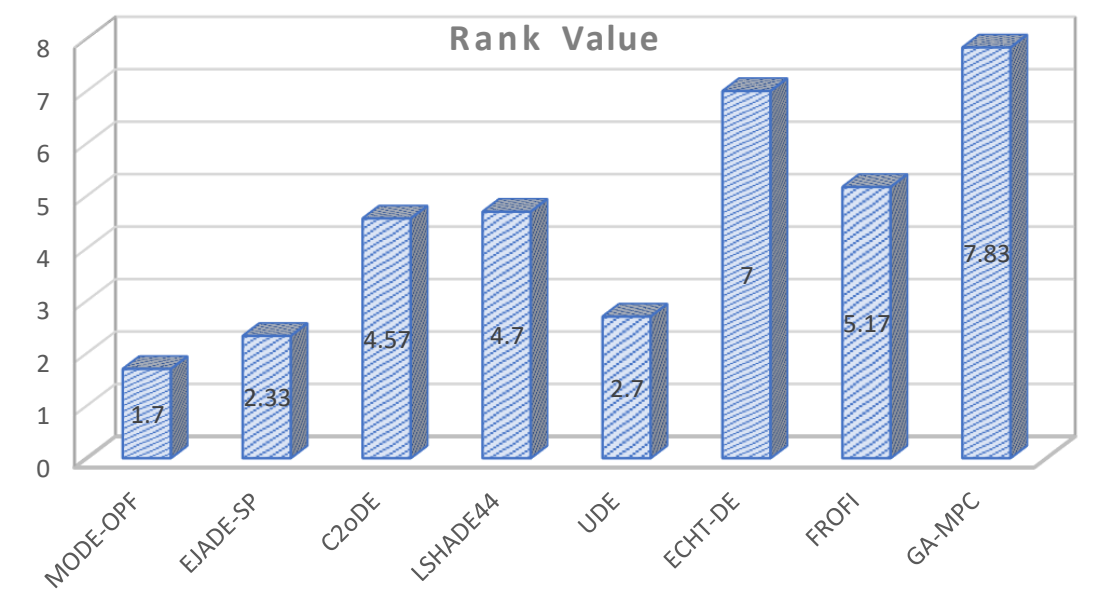

Figure 9: Case 1: Average ranking achieved by Friedman's ranking test

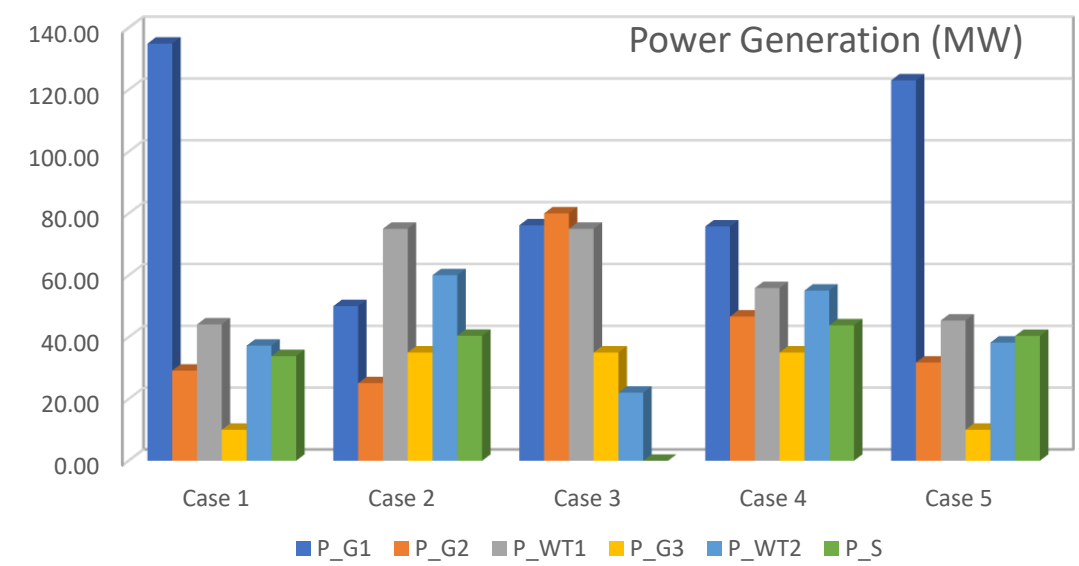

Figure 10: Power generation commands for all the generators 
results from which it is clear that the proposed MODE-OPF achieved the best average and best maximum values, while EJADE-SP obtained the best minimum and median values. Therefore, it can be concluded that the proposed solver has the capability to reduce the average of the power loss by $0.98 \%, 0.16 \%, 0.63 \%, 0.13 \%, 6.80 \%, 0.25 \%$ and $22.13 \%$, as compared to EJADE-SP, C ${ }^{2} \mathrm{ODE}$, LSHADE44, UDE, ECHT-DE, FRORI and GA-MPC, respectively. It can be also observed from Figure 16 that the real power loss is less in this objective function as compared to the other methods while operating cost has increased slightly than Case 1. Power loss has been minimized by reducing thermal power generation and supplying more renewable power at bus 5, 11 and 13, as demonstrated in Figure 10. As higher power is generated from renewable energy sources, leading to less emission as shown in Figure 18, the cumulative voltage deviation has increased.

Table 9: Case 2: Comparative summary of algorithms

\begin{tabular}{cccccc}
\hline Algorithms & Best & Median & Mean & Worst & Std. \\
\hline GA-MPC & 2.1347500 & 2.2197700 & 2.2952036 & 3.5394740 & 0.3042764 \\
EJADE-SP & $\mathbf{2 . 0 7 3 1 2 4 0 0 0}$ & $\mathbf{2 . 0 7 4 0 8 9 0 0 0}$ & 2.083661733 & 2.103725000 & 0.014396839 \\
C $^{2}$ oDE & 2.074268000 & 2.074929000 & 2.075417500 & 2.081397000 & 0.001516278 \\
LSHADE44 & 2.074141000 & 2.074187500 & 2.080185800 & 2.162454000 & 0.019715889 \\
UDE & 2.074121398 & 2.074121407 & 2.075107860 & 2.103714801 & 0.005402989 \\
ECHT-DE & 2.114171000 & 2.141488500 & 2.141869033 & 2.162325000 & 0.013077309 \\
FROFI & 2.074726206 & 2.075962943 & 2.076313577 & 2.078697183 & 0.001066128 \\
MODE-OPF & 2.073235906 & 2.074121590 & $\mathbf{2 . 0 7 3 8 5 0 0 7}$ & $\mathbf{2 . 0 7 4 1 2 7 7 9 8}$ & $\mathbf{0 . 0 0 0 4 1 0 9 6 7}$ \\
\hline
\end{tabular}

Table 10 presents the results of the Wilcoxon signed-rank test, from which it can be seen that MODE-OPF is significantly better than $\mathrm{C}^{2} \mathrm{oDE}$, LSHADE44, ECHT-DE, FRORI and GA-MPC with a p-value less than 0.05, while there is no statistical difference with EJADE-SP and UDE. Note the p-value when comparing MODE-OPF with EJADE-SP is 0.061 which is slightly greater than 0.05 . For further analysis, Friedman's rank test is conducted to rank the proposed algorithm and the rival algorithms with the result depicted in Figure 11. It is clear from this figure that MODE-OPF and UDE are ranked first, EJADE-SP is ranked second, while GA-MPC is ranked last.

Table 10: Case 2: Results obtained by the Wilcoxon test

\begin{tabular}{ccccc}
\hline Algorithms & $R^{+}$ & $R^{-}$ & Exact P-value & Dec. \\
\hline MODE-OPF vs. EJADE-SP & 323.0 & 142.0 & 0.06356 & $\approx$ \\
MODE-OPF vs. C ${ }^{2}$ oDE & 465.0 & 0.0 & $1.8626 \mathrm{E}-9$ & + \\
MODE-OPF vs. LSHADE44 & 465.0 & 0.0 & $1.8626 \mathrm{E}-9$ & + \\
MODE-OPF vs. UDE & 239.0 & 226.0 & $\geq 0.2$ & $\approx$ \\
MODE-OPF vs. ECHT-DE & 465.0 & 0.0 & $1.8626 \mathrm{E}-9$ & + \\
MODE-OPF vs. FROFI & 465.0 & 0.0 & $1.8626 \mathrm{E}-9$ & + \\
MODE-OPF vs.GA-MPC & 465.0 & 0.0 & $1.8626 \mathrm{E}-9$ & + \\
\hline
\end{tabular}

\subsection{Results for case 3}

Voltage deviation is defined as the discrepancy between nominal and real voltage. The lower the deviation of the generated voltage, the better the voltage condition of the system. Thus, optimizing (minimizing) the voltage deviation is crucial in the power network. Therefore, the objective function in this case is used to minimize the voltage deviation from the reference voltage, 1 p.u. The voltages of 24 load buses in IEEE 30-bus system can vary in between 0.95 p.u. and 1.05 p.u. Therefore, the maximum permissible voltage deviation for the cumulative approach is theoretically 1.2 p.u. (i.e., $24 \times 0.05$ p.u.) during the network operation. It can be observed from Table 18 that cumulative voltage deviation for all the cases is within permissible ranges. The voltage deviation of all the cases for 30 buses is depicted in Figure 21. It is also found that the objective function determines the minimum voltage deviation from the reference voltage, 1 p.u. as illustrated in Figure 17. The summary of the results for minimum, median, average and maximum obtained from the proposed MODE-OPF and the state-of-the-art algorithms is given in Table 11. In regards to the minimum voltage deviation, MODE-OPF is able to obtain the best minimum value similar to both EJADE-SP and 


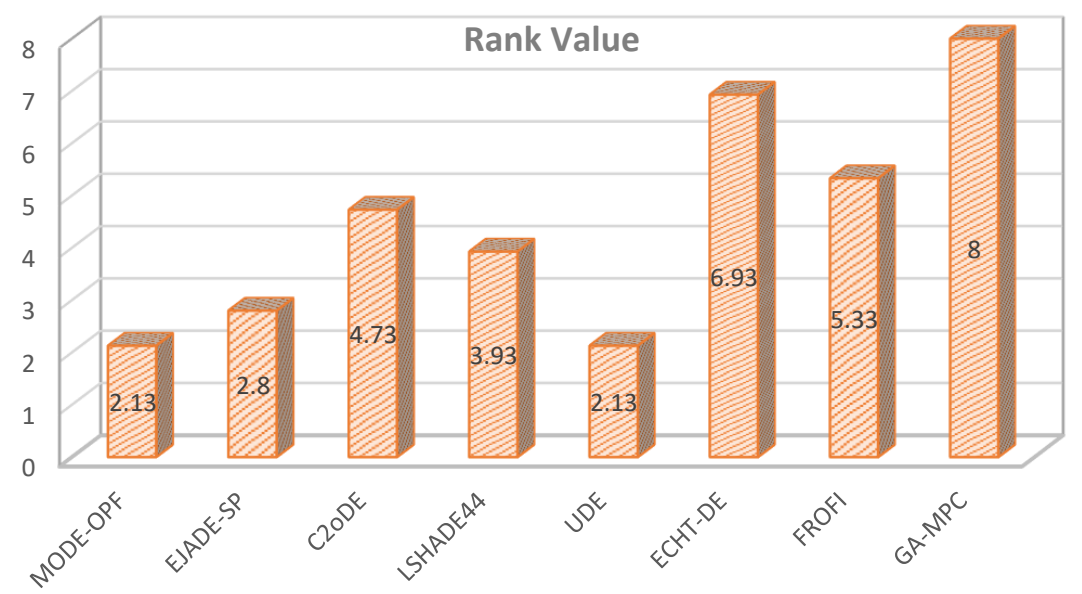

Figure 11: Case 2: Average ranking achieved by Friedman's ranking test

LSHADE44. Additionally, the proposed MODE-OPF obtains the best median, the best average and the best maximum voltage deviation values.

Table 11: Case 3: Comparative summary of algorithms

\begin{tabular}{cccccc}
\hline Algorithms & Best & Median & Mean & Worst & Std. \\
\hline GA-MPC & 0.3756540 & 0.3770276 & 0.3790136 & 0.4023681 & 0.0062173 \\
EJADE-SP & $\mathbf{0 . 3 7 5 2 2 9 7}$ & 0.3757606 & 0.3757257 & 0.3757625 & 0.0001348 \\
C $^{2}$ oDE & 0.3752298 & 0.3752486 & 0.3754349 & 0.3757626 & 0.0002506 \\
LSHADE44 & $\mathbf{0 . 3 7 5 2 2 9 7}$ & 0.3754566 & 0.3754991 & $\mathbf{0 . 3 7 5 7 6 2 0}$ & 0.0002544 \\
UDE & 0.3755108 & 0.3755807 & 0.3755746 & 0.3755817 & 0.0000177 \\
ECHT-DE & 0.3758577 & 0.3760752 & 0.3760738 & 0.3763896 & 0.0001167 \\
FROFI & 0.3752349 & 0.3756336 & 0.3755222 & 0.3757691 & 0.0002483 \\
MODE-OPF & $\mathbf{0 . 3 7 5 2 2 9 7}$ & $\mathbf{0 . 3 7 5 2 2 9 7}$ & $\mathbf{0 . 3 7 5 4 0 6 8}$ & $\mathbf{0 . 3 7 5 7 6 2 0}$ & $\mathbf{0 . 0 0 0 2 5 4 7}$ \\
\hline
\end{tabular}

Table 12 presents the results obtained from the Wilcoxon signed-rank test from which the proposed MODE-OPF is significantly superior to EJADE-SP, LSHADE44, UDE, ECHT-DE, FROFI and GA-MPC with very small p-values less than 0.05. The proposed MODE-OPF has no significant difference with $\mathrm{C}^{2} \mathrm{oDE}$, but a very small P-value less than 0.1 (0.083). For further analysis, we conducted the Friedman rank test to rank the proposed algorithms with the state-of-the-art algorithms. Figure 12 depicts the result obtained from the Friedman test, from which the proposed MODE-OPF is ranked first followed by EJADE-SP then UDE, while GA-MPC has the last rank.

Table 12: Case 3: Results obtained by the Wilcoxon test for algorithm Proposed

\begin{tabular}{ccccc}
\hline Algorithms & $R^{+}$ & $R^{-}$ & Exact P-value & Dec. \\
\hline MODE-OPF vs. EJADE-SP & 426.0 & 39.0 & $1.3966 \mathrm{E}-5$ & + \\
MODE-OPF vs. C ${ }^{2}$ oDE & 316.0 & 149.0 & 0.08794 & $\approx$ \\
MODE-OPF vs. LSHADE44 & 333.0 & 132.0 & 0.03842 & + \\
MODE-OPF vs. UDE & 410.0 & 55.0 & $9.902 \mathrm{E}-5$ & + \\
MODE-OPF vs. ECHT-DE & 465.0 & 0.0 & $1.8626 \mathrm{E}-9$ & + \\
MODE-OPF vs. FROFI & 370.0 & 95.0 & 0.003744 & + \\
MODE-OPF vs. GA-MPC & 465.0 & 0.0 & $1.8626 \mathrm{E}-9$ & + \\
\hline
\end{tabular}




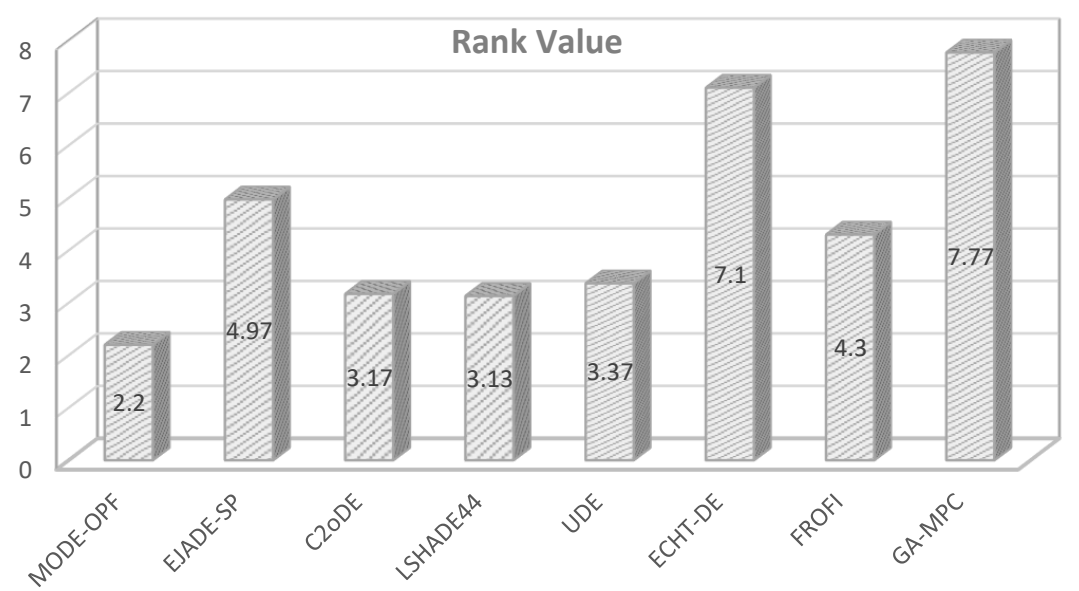

Figure 12: Case 3: Average ranking achieved by Friedman's ranking test

\subsection{Results for Case 4}

As power and electricity are produced from conventional sources (for example, fossil fuels), toxic gases are released into the air. Indeed, because of global warming and deforestation, emissions have recently gained growing attention. Therefore it is very important to reduce and minimize the emission in a power system network. As a result, the objective function of Case 4 is used to minimize emission. The summary of the results for minimum, median, maximum and average obtained from MODE-OPF and the competitive algorithms is presented in Table 13. As clear from Table 13, the proposed algorithm obtains the second minimum emission value after EJADE-SP with a very small difference (0.0000002). For the median, average and maximum emission values, the proposed MODE-OPF is able to obtain the best values similar to EJADE-SP. Figure 18 demonstrates the emission of all the five case studies from where it is clear that the objective function reduces the emission by utilizing renewable energy generation that is slightly less than Case 2. As higher renewable energy utilization in Case 2, 3 and 4, the lower emission is observed as compared to Case 1 and Case 5.

\begin{tabular}{cccccc}
\multicolumn{7}{c}{ Table 13: Case 4: Comparative summary of the algorithms } \\
\hline Algorithms & Best & Median & Mean & Worst & Std. \\
\hline GA-MPC & 0.0958581 & 0.0960947 & 0.0971113 & 0.1131335 & 0.0038195 \\
EJADE-SP & $\mathbf{0 . 0 9 5 8 3 2 5}$ & $\mathbf{0 . 0 9 5 8 3 2 7}$ & $\mathbf{0 . 0 9 5 8 3 2 7}$ & $\mathbf{0 . 0 9 5 8 3 2 9}$ & 0.0000001 \\
C $^{2}$ oDE & 0.0958328 & 0.0958355 & 0.0958364 & 0.0958449 & 0.0000033 \\
LSHADE44 & 0.0958327 & 0.0958330 & 0.0958338 & 0.0958394 & 0.0000016 \\
UDE & 0.0958327 & 0.0958328 & 0.0958329 & 0.0958337 & 0.0000002 \\
ECHT-DE & 0.0958416 & 0.0958591 & 0.0958633 & 0.0959138 & 0.0000162 \\
FROFI & 0.0958373 & 0.0958480 & 0.0958478 & 0.0958698 & 0.0000082 \\
MODE-OPF & 0.0958327 & $\mathbf{0 . 0 9 5 8 3 2 7}$ & $\mathbf{0 . 0 9 5 8 3 2 7}$ & $\mathbf{0 . 0 9 5 8 3 2 9}$ & $\mathbf{0 . 0 0 0 0 0 0 0}$ \\
\hline
\end{tabular}

For further analysis, we have conducted the Wilcoxon signed-rank test to see if there is a significant difference between the proposed algorithm (MODE-OPF) and other algorithms, with the results presented in Table 14. It can be concluded from this table that MODE-OPF is significantly better than $\mathrm{C}^{2}$ oDE, LSHADE44, UDE, ECHT-DE, FROFI and GA-MPC. However, there is no significant difference between the proposed algorithm and EJADE-SP. For a more statistical test, we have conducted the Friedman rank test in order to rank all the competing algorithms, with the results shown in Figure 13. It is clear that MODE-OPF is ranked first, followed by EJADE-SP in the second position, then UDE in the thied position, while GA-MPC comes in the last position. 
Table 14: Case 4: Results obtained by the Wilcoxon test for algorithm Proposed

\begin{tabular}{ccccc}
\hline Algorithms & $R^{+}$ & $R^{-}$ & Exact P-value & Dec. \\
\hline MODE-OPF vs. EJADE-SP & 231.0 & 234.0 & $\geq 0.2$ & $\approx$ \\
MODE-OPF vs. C ${ }^{2}$ oDE & 465.0 & 0.0 & $1.8626 \mathrm{E}-9$ & + \\
MODE-OPF vs. LSHADE44 & 417.0 & 18.0 & $9.424 \mathrm{E}-7$ & + \\
MODE-OPF vs. UDE & 463.5 & 1.5 & $4.657 \mathrm{E}-9$ & + \\
MODE-OPF vs. ECHT-DE & 465.0 & 0.0 & $1.8626 \mathrm{E}-9$ & + \\
MODE-OPF vs. FROFI & 465.0 & 0.0 & $1.8626 \mathrm{E}-9$ & + \\
MODE-OPF vs. GA-MPC & 465.0 & 0.0 & $1.8626 \mathrm{E}-9$ & + \\
\hline
\end{tabular}

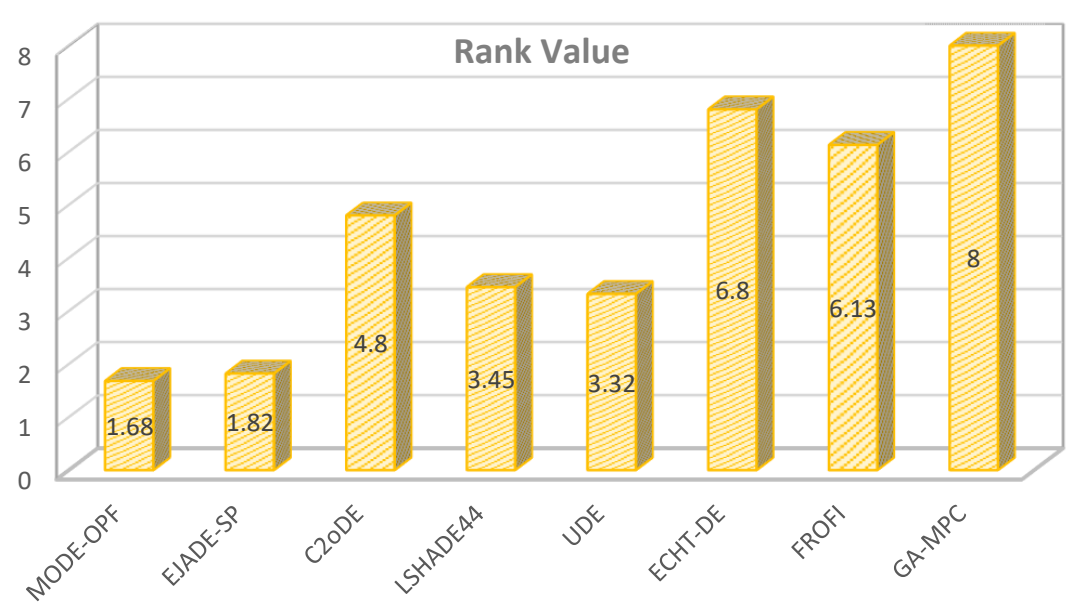

Figure 13: Case 4: Average ranking achieved by Friedman's ranking test

\subsubsection{Results of case 5}

In this situation, we considered emission and generation costs. The summary of the statistical results are presented in Table 16, from which the proposed MODE-OPF has the ability to obtain better results than all other rival algorithms for best, median, mean and worst objective function values. EJADE-SP is able to obtain the best minimum value, but not the best for other criteria. This proves the robustness of the proposed MODE-OPF. Therefore, it can be concluded that the proposed solver has the capability to reduce the average of both emission and total generation cost by $1.1 \%$, $0.83 \%, 2.70 \%, 1.32 \%, 8.56 \%, 1.1 \%$ and $28.95 \%$, as compared to EJADE-SP, $\mathrm{C}^{2} \mathrm{ODE}$, LSHADE44, UDE, ECHT-DE, FRORI and GA-MPC, respectively. It can be observed from Table 19 that the minimum operating cost is achieved by this case study, which is $\$ 811.23$. Therefore, we can conclude that combining the two cases (i.e., 1 and 4 ) provides the lowest operational cost with low environmental pollution.

\begin{tabular}{cccccc}
\multicolumn{7}{c}{ Table 15: Case 5: Comparative summary of the algorithms } \\
\hline Algorithms & Best & Median & Mean & Worst & Std. \\
\hline GA-MPC & 811.260700 & 812.453150 & 813.587130 & 827.368900 & 4.239141 \\
EJADE-SP & $\mathbf{8 1 1 . 2 2 6 9 2 0}$ & 811.227010 & 811.242495 & 811.521965 & 0.053385 \\
C $^{2}$ oDE & 811.230000 & 811.240800 & 811.239783 & 811.245200 & 0.005216 \\
LSHADE44 & 811.227000 & 811.243900 & 811.258470 & 811.615700 & 0.074488 \\
UDE & 811.227002 & 811.243869 & 811.244706 & 811.367322 & 0.024636 \\
ECHT-DE & 811.284000 & 811.311950 & 811.317110 & 811.401000 & 0.028397 \\
FROFI & 811.234212 & 811.243417 & 811.242496 & 811.248956 & 0.004073 \\
MODE-OPF & $\mathbf{8 1 1 . 2 2 6 9 2 0}$ & $\mathbf{8 1 1 . 2 2 7 0 0 2}$ & $\mathbf{8 1 1 . 2 3 1 5 0 0}$ & $\mathbf{8 1 1 . 2 4 3 8 6 9}$ & 0.007587 \\
\hline
\end{tabular}

Considering the Wilcoxon's signed-rank test, as in the last column of Table 16, the proposed MODE-OPF is signif- 
icantly better than all other competing algorithms with p-values less than 0.05 . Furthermore, referring to Friedman's ranking test as depicted in Figure 14, the proposed algorithm is ranked first, EJADE-SP ranked second and UDE ranked third, while GA-MPC ranked last.

Table 16: Case 5: Results obtained by the Wilcoxon test

\begin{tabular}{ccccc}
\hline Algorithms & $R^{+}$ & $R^{-}$ & Exact P-value & Dec. \\
\hline MODE-OPF vs. EJADE-SP & 332.0 & 133.0 & 0.04048 & + \\
MODE-OPF vs. C ${ }^{2}$ oDE & 393.0 & 72.0 & $5.548 \mathrm{E}-4$ & + \\
MODE-OPF vs. LSHADE44 & 422.0 & 43.0 & $2.366 \mathrm{E}-5$ & + \\
MODE-OPF vs. UDE & 384.0 & 51.0 & $1.2406 \mathrm{E}-4$ & + \\
MODE-OPF vs. ECHT-DE & 465.0 & 0.0 & $1.8626 \mathrm{E}-9$ & + \\
MODE-OPF vs. FROFI & 442.0 & 23.0 & $1.192 \mathrm{E}-6$ & + \\
MODE-OPF vs. GA-MPC & 465.0 & 0.0 & $1.8626 \mathrm{E}-9$ & + \\
\hline
\end{tabular}

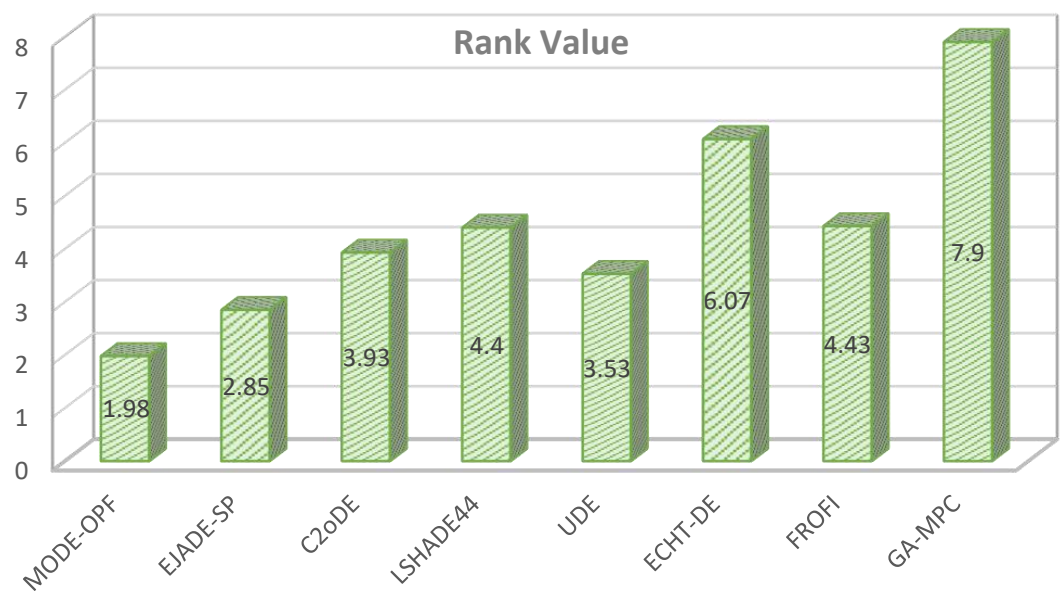

Figure 14: Case 5: Average ranking achieved by Friedman's ranking test

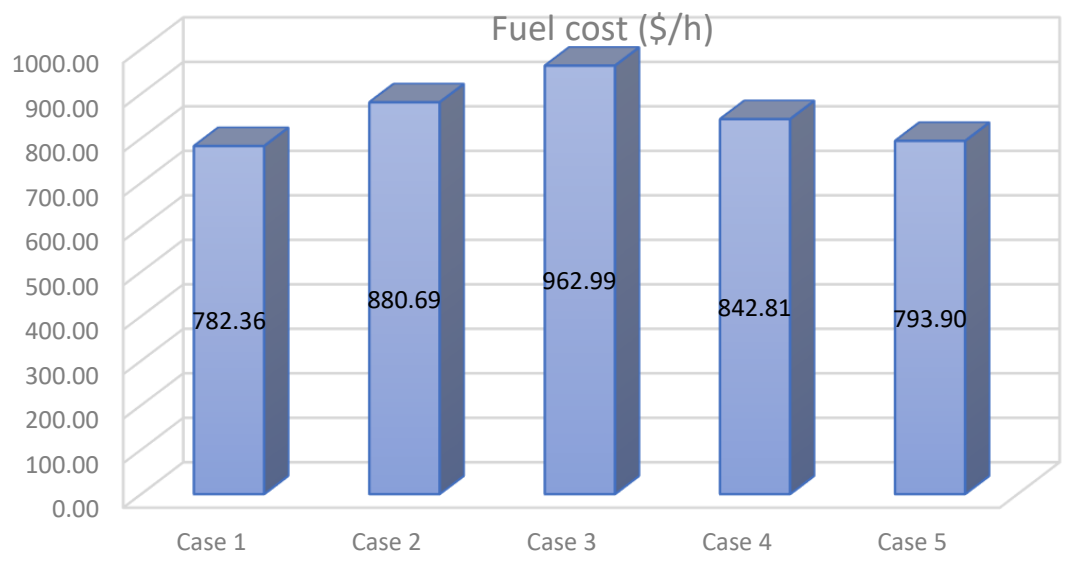

Figure 15: Fuel costs of all the cases

\subsubsection{Convergence, computational time and voltage curves}

The efficiency of the proposed MODE-OPF is further assessed by plotting the convergence graphs for all cases, as depicted in Figure 20. From Figure 20, it is clear that the proposed algorithm converges faster than all other 


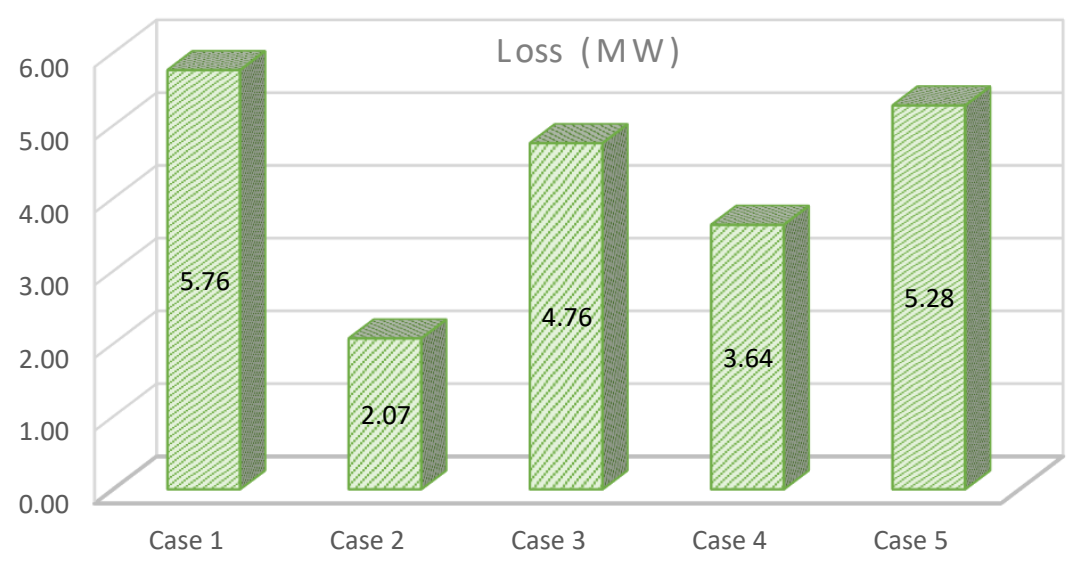

Figure 16: Real power loss of the network

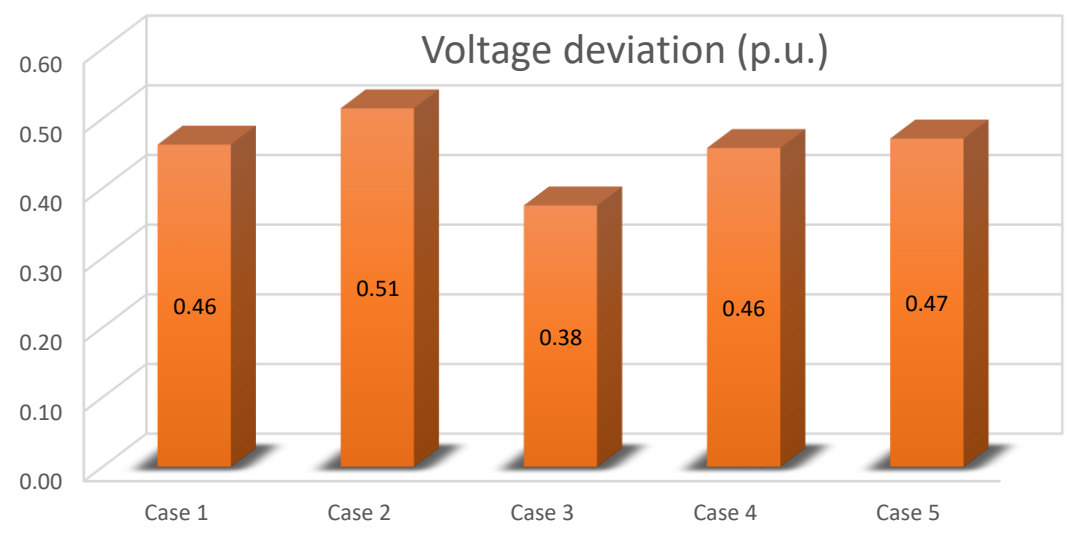

Figure 17: Cumulative voltage drop of the network

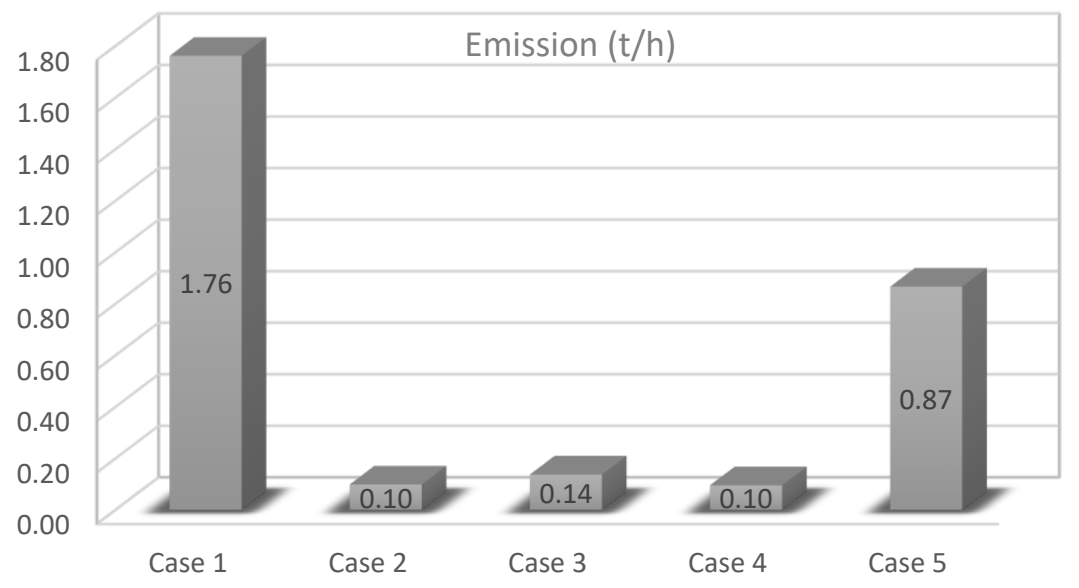

Figure 18: Emission of generators for all the cases 


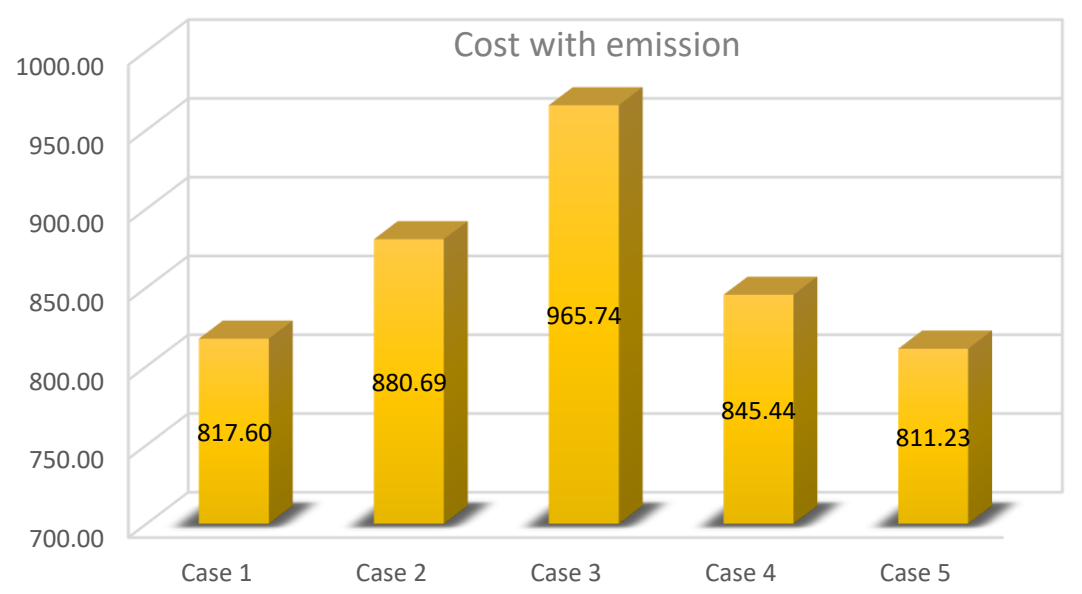

Figure 19: Generation cost and emission cost together

algorithms. For example, in case of 1 , the proposed algorithm (MODE-OPF) has the fastest convergence followed by EJADE-SP, then $\mathrm{C}^{2} \mathrm{oDE}$. Again, we consider case 3, MODE-OPF has the fastest convergence, followed by UDE and then ECHT-DE.

Also, as a further comparison, the average computational running time over the 30 runs for all competing algorithms are recorded in Table 17. It can be concluded from Table 17 that the average computational time of the proposed MODE-OPF is smaller than the average computational time of all other algorithms. The proposed algorithm is able to save the time up to $25.57 \%, 30.88 \%, 49.4 \%, 37.45 \%$ and $34.64 \%$ for Case 1, Case 2, Case 3, Case 4 and Case 5, respectively.

Table 17: Average computational time in seconds for the five different cases

\begin{tabular}{ccccccccc}
\hline Algorithms & MODE-OPF & EJADE-SP & C $^{2}$ oDE & LSHADE44 & UDE & ECHT-DE & FROFI & GA-MPC \\
\hline Case 1 & $\mathbf{1 6 4 . 2 9 7}$ & 204.299 & 208.356 & 206.147 & 208.329 & 220.742 & 171.064 & 188.214 \\
Case 2 & $\mathbf{1 6 1 . 0 0 0}$ & 174.838 & 222.336 & 199.094 & 232.918 & 222.410 & 174.422 & 222.013 \\
Case 3 & $\mathbf{1 4 2 . 9 8 4}$ & 282.758 & 272.250 & 266.123 & 203.543 & 260.943 & 229.972 & 198.833 \\
Case 4 & $\mathbf{1 5 1 . 5 7 8}$ & 173.916 & 239.993 & 242.319 & 191.514 & 229.243 & 193.508 & 186.436 \\
Case 5 & $\mathbf{1 4 4 . 3 2 0}$ & 193.455 & 203.926 & 220.823 & 158.129 & 213.330 & 158.117 & 161.354 \\
\hline
\end{tabular}

In a power system, the load bus voltage is a vital constraint. So, its values should be retained within a specific range, i.e., the values should be in [0.95p.u, 1.05p.u] [12]. Figure 21 depicts the voltage profiles of load busses for the different five cases (case 1 to case 5). It is clear from Figure 21 that the load bus voltage always satisfies the requirements independent of any considered cases.

\subsection{Comparison with the state-of-the-art algorithms for IEEE 118-bus system}

In this section, the performance of the proposed MODE-OPF is tested on the larger system (IEEE 118-bus system), while the objective is to minimize fuel cost (case 1) and power loss (case 2). For keeping the length of this manuscript within acceptable range, we have deliberately avoided the performance demonstration of MODE-OPF for this larger dataset while considering other objectives (i.e., cases 3 to 5). The calculation details of these objective functions and their associated constrained are presented in Subsection 2.3 and Subsection 2.4, respectively. MODE-OPF was run 30 times for 100,000 fitness evaluations, with population size equal to 75 . Note, for a fair comparison all the compared algorithms were run to the same number of fitness evaluations $(100,000)$ and the population size was set to a value equal to 75. The ranges of control and state variables of the IEEE 118-bus system are presented in Table 19.

Tables 20 and 21 present the values of the control variables and the calculated parameters of the best objective function values produced in 30 runs for fuel cost (case 1) and power loss (case 2), respectively. For case 1, the obtained value of fuel cost is $134993.01 \$ / \mathrm{h}$. For case 2, the obtained real power loss from MODE-OPF is $16.875146 \mathrm{MW}$. 


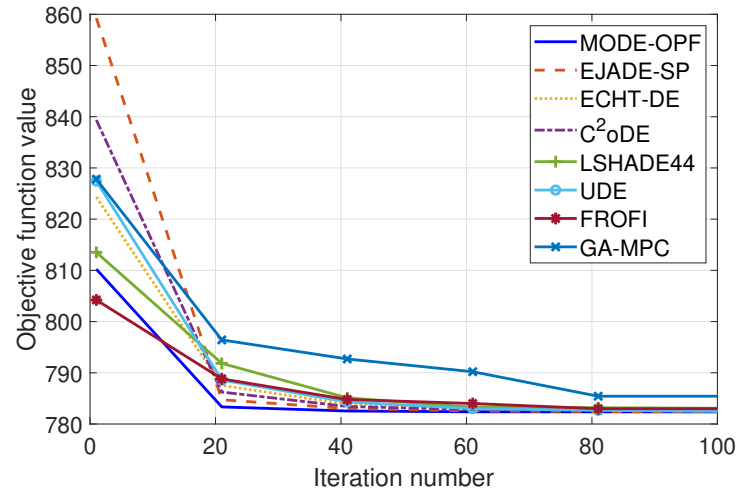

(a)

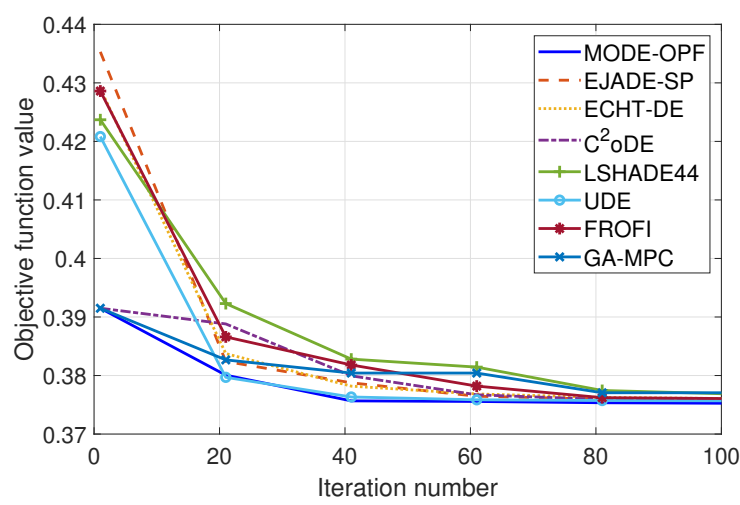

(c)

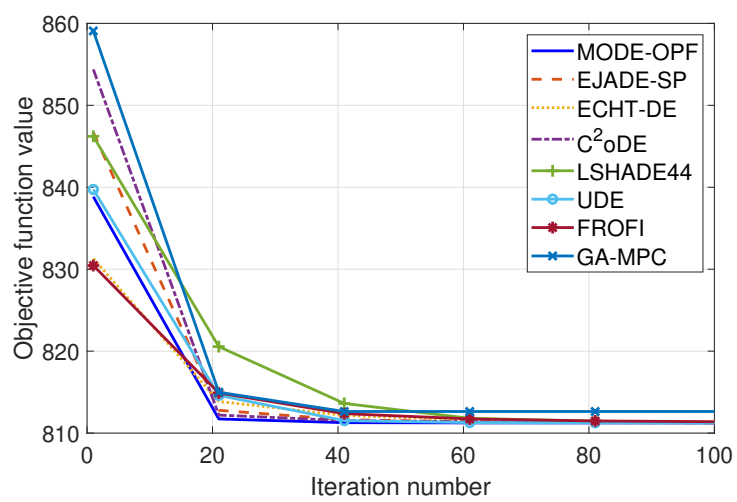

(e)

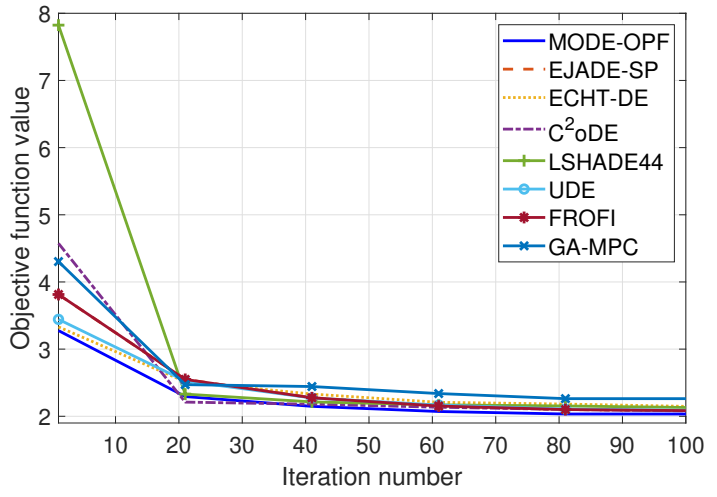

(b)

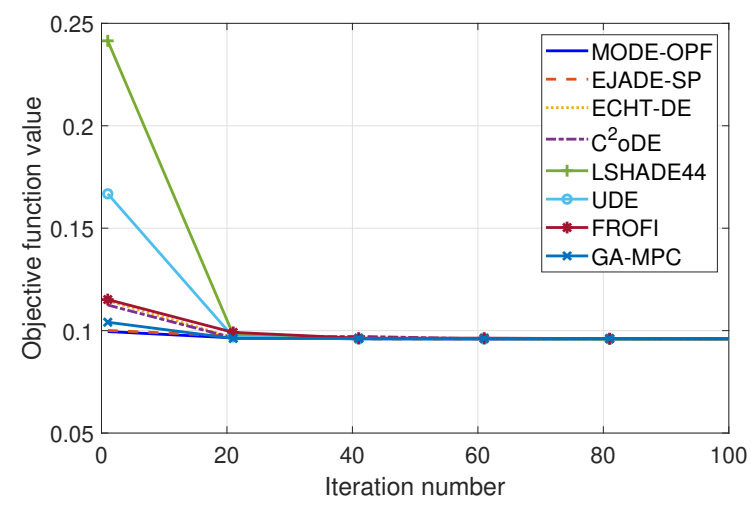

(d)

Figure 20: Convergence graphs of algorithms compared: (a) Case 1; (b) Case 2; (c) Case 3; (d) Case 4; and (e) Case 5 


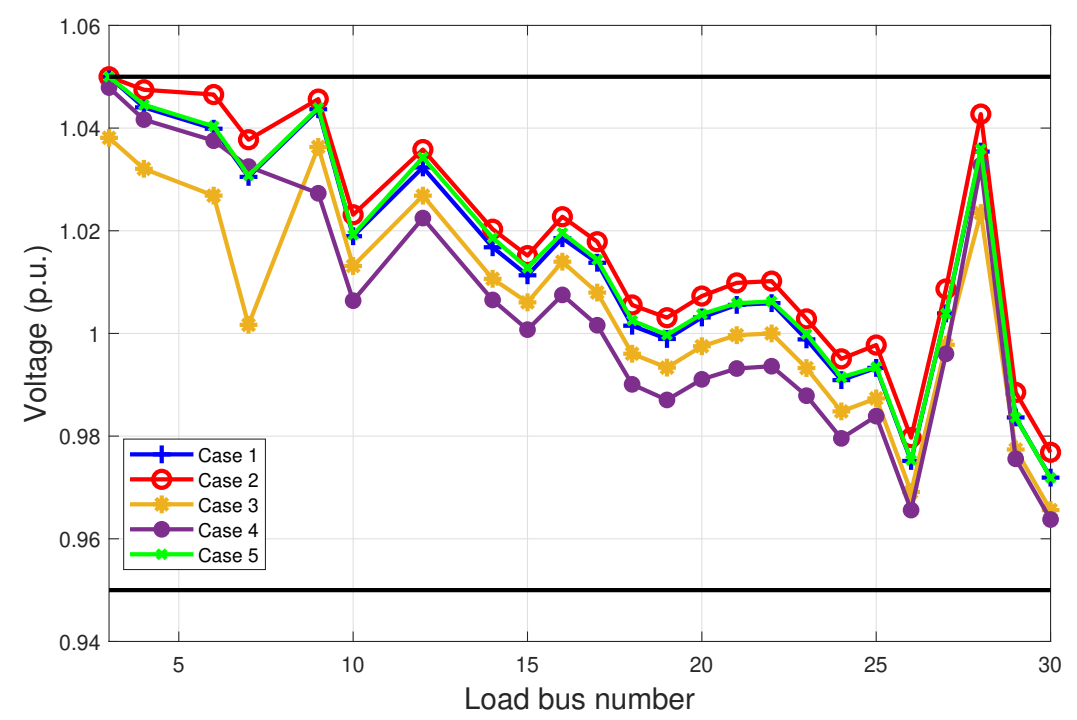

Figure 21: Voltages of all buses for the best obtained solutions for all five cases

Table 18: Optimal results obtained from MODE-OPF of the IEEE-30 bus system for Cases 1-5

\begin{tabular}{|c|c|c|c|c|c|c|c|}
\hline \multirow{2}{*}{ Control Variable } & \multicolumn{2}{|c|}{ Bounds of Variables } & \multirow{2}{*}{ Case 1} & \multirow{2}{*}{ Case 2} & \multirow{2}{*}{ Case 3} & \multirow{2}{*}{ Case 4} & \multirow{2}{*}{ Case 5} \\
\hline & Min & Max & & & & & \\
\hline$P_{G_{1}}$ & 30 & 140 & 134.908 & 50.000 & 76.145 & 75.753 & 123.027 \\
\hline$P_{G_{2}}$ & 20 & 80 & 29.130 & 25.068 & 80.000 & 46.634 & 31.746 \\
\hline$P_{G_{3}}$ & 0 & 75 & 44.085 & 75.000 & 75.000 & 55.837 & 45.33 \\
\hline$P_{G_{4}}$ & 10 & 35 & 10.00 & 35.000 & 35.000 & 35.000 & 10.000 \\
\hline$P_{G_{5}}$ & 0 & 60 & 37.202 & 60.000 & 22.013 & 55.009 & 38.194 \\
\hline$P_{G_{6}}$ & 0 & 50 & 33.838 & 40.406 & 0.000 & 43.802 & 40.371 \\
\hline$V_{G_{1}}$ & 0.95 & 1.10 & 1.072 & 1.058 & 1.061 & 1.072 & 1.070 \\
\hline$V_{G_{2}}$ & 0.95 & 1.10 & 1.058 & 1.053 & 1.100 & 1.027 & 1.057 \\
\hline$V_{G_{5}}$ & 0.95 & 1.10 & 1.036 & 1.044 & 0.951 & 1.044 & 1.036 \\
\hline$V_{G_{8}}$ & 0.95 & 1.10 & 1.040 & 1.050 & 1.100 & 1.038 & 1.040 \\
\hline$V_{G_{11}}$ & 0.95 & 1.10 & 1.099 & 1.097 & 1.100 & 1.065 & 1.100 \\
\hline$V_{G_{13}}$ & 0.95 & 1.10 & 1.054 & 1.059 & 1.100 & 1.042 & 1.057 \\
\hline$Q_{G_{1}}$ & -20 & 150 & -1.878 & -5.096 & -8.584 & 26.150 & -2.794 \\
\hline$Q_{G_{2}}$ & -20 & 60 & 13.299 & 7.106 & 60.000 & -20.000 & 12.181 \\
\hline$Q_{G_{3}}$ & -15 & 40 & 23.170 & 20.815 & -30.000 & 32.731 & 22.988 \\
\hline$Q_{G_{4}}$ & -30 & 35 & 35.000 & 37.903 & 40.000 & 36.729 & 35.120 \\
\hline$Q_{G_{5}}$ & -25 & 30 & 30.000 & 30.000 & 30.000 & 22.013 & 30.000 \\
\hline$Q_{G_{6}}$ & -20 & 25 & 17.216 & 18.596 & 25.000 & 15.995 & 18.196 \\
\hline Total fuel cost $(\$ / h)$ & & & 782.35930 & 880.69180 & 962.98720 & 842.81070 & 793.8983 \\
\hline Wind power cost & & & 248.29260 & 464.62960 & 329.54980 & 361.88210 & 256.1482 \\
\hline Solar power cost & & & 91.39878 & 113.96730 & 45.64850 & 126.66270 & 113.8466 \\
\hline Thermal generator cost & & & 442.66790 & 302.09490 & 587.78880 & 354.26590 & 423.9035 \\
\hline Real power loss (MW) & & & 5.76321 & 2.07324 & 4.75823 & 3.63551 & 5.2767 \\
\hline Cumulative voltage deviation & & & 0.46252 & 0.51470 & 0.37523 & 0.45764 & 0.4713 \\
\hline Emission (ton/h) & & & 1.76193 & 0.09894 & 0.13763 & 0.09583 & 0.8664 \\
\hline Total fuel cost +Emission & & & 817.59798 & 880.69180 & 965.73975 & 845.44365 & 811.2270 \\
\hline
\end{tabular}


Table 19: Lower and upper bounds for IEEE 118-bus system.

\begin{tabular}{|c|c|c|c|c|c|c|c|c|c|}
\hline $\begin{array}{c}\text { Control } \\
\text { variables }\end{array}$ & Range (MW) & $\begin{array}{c}\text { Control } \\
\text { variables }\end{array}$ & Range (MW) & $\begin{array}{c}\text { Control } \\
\text { variables }\end{array}$ & Range (p.u.) & $\begin{array}{c}\text { Control } \\
\text { variables }\end{array}$ & Range (p.u.) & $\begin{array}{c}\text { Control } \\
\text { variables }\end{array}$ & Range \\
\hline PG1 (MW) & $30-100$ & PG65 & $147.3-491$ & $V \mathrm{G} 1$ (MW) & $0.95-1.1$ & $V$ G65 & $0.95-1.1$ & QC5 (MVAr) & $0-25$ \\
\hline PG4 & $30-100$ & PG66 & $147.6-492$ & $V \mathrm{G} 4$ & $0.95-1.1$ & VG66 & $0.95-1.1$ & QC34 (MVAr) & $0-25$ \\
\hline PG6 & $30-100$ & $P \mathrm{G} 70$ & $30-100$ & VG6 & $0.95-1.1$ & $V G 69$ & $0.95-1.1$ & QC37 (MVAr) & $0-25$ \\
\hline PG8 & $30-100$ & PG72 & $30-100$ & $V \mathrm{G} 8$ & $0.95-1.1$ & VG70 & $0.95-1.1$ & QC44 (MVAr) & $0-25$ \\
\hline$P G 10$ & $165-550$ & PG73 & $30-100$ & $V G 10$ & $0.95-1.1$ & $V G 72$ & $0.95-1.1$ & QC45 (MVAr) & $0-25$ \\
\hline PG12 & $55.5-185$ & PG74 & $30-100$ & $V G 12$ & $0.95-1.1$ & $V G 73$ & $0.95-1.1$ & QC46 (MVAr) & $0-25$ \\
\hline PG15 & $30-100$ & PG76 & $30-100$ & $V G 15$ & $0.95-1.1$ & $V G 74$ & $0.95-1.1$ & QC48 (MVAr) & $0-25$ \\
\hline PG18 & $30-100$ & PG77 & $30-100$ & $V G 18$ & $0.95-1.1$ & $V G 76$ & $0.95-1.1$ & QC74 (MVAr) & $0-25$ \\
\hline PG19 & $30-100$ & PG80 & $173.1-577$ & $V G 19$ & $0.95-1.1$ & $V G 77$ & $0.95-1.1$ & QC79 (MVAr) & $0-25$ \\
\hline$P G 24$ & $30-100$ & PG85 & $30-100$ & $V G 24$ & $0.95-1.1$ & $V G 80$ & $0.95-1.1$ & QC82 (MVAr) & $0-25$ \\
\hline PG25 & $96-320$ & PG87 & $31.2-104$ & $V G 25$ & $0.95-1.1$ & $V G 85$ & $0.95-1.1$ & QC83 (MVAr) & $0-25$ \\
\hline PG26 & $124.2-414$ & PG89 & $212.1-707$ & $V G 26$ & $0.95-1.1$ & VG87 & $0.95-1.1$ & QC105 (MVAr) & $0-25$ \\
\hline PG27 & $30-100$ & PG90 & $30-100$ & $V G 27$ & $0.95-1.1$ & $V G 89$ & $0.95-1.1$ & QC107 (MVAr) & $0-25$ \\
\hline PG31 & $32.1-107$ & PG91 & $30-100$ & $V G 31$ & $0.95-1.1$ & $V G 90$ & $0.95-1.1$ & QC110 (MVAr) & $0-25$ \\
\hline PG32 & $30-100$ & PG92 & $30-100$ & $V G 32$ & $0.95-1.1$ & $V G 91$ & $0.95-1.1$ & $T 8$ (p.u.) & $0.9-1.1$ \\
\hline PG34 & $30-100$ & PG99 & $30-100$ & $V G 34$ & $0.95-1.1$ & $V G 92$ & $0.95-1.1$ & T32 (p.u.) & $0.9-1.1$ \\
\hline PG36 & $30-100$ & $P G 100$ & $105.6-352$ & $V G 36$ & $0.95-1.1$ & $V G 99$ & $0.95-1.1$ & $T 36$ (p.u.) & $0.9-1.1$ \\
\hline PG40 & $30-100$ & $P G 103$ & $42-140$ & $V G 40$ & $0.95-1.1$ & $V G 100$ & $0.95-1.1$ & $T 51$ (p.u.) & $0.9-1.1$ \\
\hline PG42 & $30-100$ & PG104 & $30-100$ & $V G 42$ & $0.95-1.1$ & $V G 103$ & $0.95-1.1$ & $T 93$ (p.u.) & $0.9-1.1$ \\
\hline PG46 & $35.7-119$ & PG105 & $30-100$ & $V G 46$ & $0.95-1.1$ & VG104 & $0.95-1.1$ & $T 95$ (p.u.) & $0.9-1.1$ \\
\hline PG49 & $91.2-304$ & PG107 & $30-100$ & $V G 49$ & $0.95-1.1$ & $V G 105$ & $0.95-1.1$ & $T 102$ (p.u.) & $0.9-1.1$ \\
\hline PG54 & $44.4-148$ & PG110 & $30-100$ & $V G 54$ & $0.95-1.1$ & $V G 107$ & $0.95-1.1$ & $T 107$ (p.u.) & $0.9-1.1$ \\
\hline PG55 & $30-100$ & PG111 & $40.8-136$ & $V G 55$ & $0.95-1.1$ & $V \mathrm{G} 110$ & $0.95-1.1$ & $T 127$ (p.u.) & $0.9-1.1$ \\
\hline PG56 & $30-100$ & PG112 & $30-100$ & VG56 & $0.95-1.1$ & $V G 111$ & $0.95-1.1$ & PG69 (MW) & $0-805.2$ \\
\hline PG59 & $76.5-255$ & PG113 & $30-100$ & $V G 59$ & $0.95-1.1$ & VG112 & $0.95-1.1$ & & \\
\hline PG61 & $78-260$ & PG116 & $30-100$ & $V G 61$ & $0.95-1.1$ & VG113 & $0.95-1.1$ & & \\
\hline PG62 & $30-100$ & & & $V G 62$ & $0.95-1.1$ & $V G 116$ & $0.95-1.1$ & & \\
\hline
\end{tabular}

Table 20: Simulation results obtained from MODE-OPF algorithm for case 1 of IEEE 118-bus system.

\begin{tabular}{|c|c|c|c|c|c|c|c|c|c|}
\hline $\begin{array}{c}\text { Control } \\
\text { variables }\end{array}$ & Values (MW) & $\begin{array}{c}\text { Control } \\
\text { variables }\end{array}$ & Values (MW) & $\begin{array}{c}\text { Control } \\
\text { variables }\end{array}$ & Values (p.u.) & $\begin{array}{c}\text { Control } \\
\text { variables }\end{array}$ & Values (p.u.) & $\begin{array}{c}\text { Control } \\
\text { variables }\end{array}$ & Values \\
\hline PG1 (MW) & 30.000301 & PG65 & 290.007900 & $V$ G1 (MW) & 1.0105823 & $V \mathrm{G} 65$ & 1.0583671 & QC5 (MVAr) & 2.28054 \\
\hline PG4 & 30.000096 & PG66 & 289.808350 & $V \mathrm{G} 4$ & 1.0385693 & VG66 & 1.0721225 & QC34 (MVAr) & 5.92327 \\
\hline PG6 & 30.000071 & $P$ G70 & 30.002357 & $V \mathrm{G} 6$ & 1.0325120 & $V G 69$ & 1.0544147 & QC37 (MVAr) & 0.01109 \\
\hline PG8 & 30.000000 & PG72 & 30.003546 & $V \mathrm{G} 8$ & 1.0443294 & $V G 70$ & 1.0395854 & QC44 (MVAr) & 6.96712 \\
\hline PG10 & 315.648560 & PG73 & 30.022168 & $V$ G10 & 1.0443034 & $V G 72$ & 1.0456533 & $Q \mathrm{C} 45$ (MVAr) & 19.95665 \\
\hline PG12 & 67.610082 & PG74 & 30.004690 & $V \mathrm{G} 12$ & 1.0247451 & $V \mathrm{G} 73$ & 1.0423548 & QC46 (MVAr) & 0.90691 \\
\hline PG15 & 30.002392 & PG76 & 30.001196 & $V$ G15 & 1.0230688 & $V G 74$ & 1.0248496 & QC48 (MVAr) & 7.61586 \\
\hline PG18 & 30.000116 & PG77 & 30.000021 & $V G 18$ & 1.0252138 & $V \mathrm{G} 76$ & 1.0030046 & QC74 (MVAr) & 24.99988 \\
\hline PG19 & 30.000657 & PG80 & 349.199090 & $V G 19$ & 1.0222603 & $V G 77$ & 1.0269050 & QC79 (MVAr) & 24.95416 \\
\hline$P G 24$ & 30.003568 & PG85 & 30.000795 & $V \mathrm{G} 24$ & 1.0381604 & $V \mathrm{G} 80$ & 1.0349583 & QC82 (MVAr) & 24.99059 \\
\hline$P G 25$ & 152.602370 & PG87 & 31.200575 & $V G 25$ & 1.0537238 & $V G 85$ & 1.0464296 & QC83 (MVAr) & 4.35272 \\
\hline PG26 & 219.960740 & PG89 & 383.557890 & $V G 26$ & 1.0807804 & VG87 & 1.0670962 & QC105 (MVAr) & 18.00007 \\
\hline$P G 27$ & 30.000013 & PG90 & 30.000020 & $V G 27$ & 1.0214670 & $V G 89$ & 1.0559942 & QC107 (MVAr) & 7.52475 \\
\hline PG31 & 32.100013 & PG91 & 30.003381 & $V G 31$ & 1.0166141 & $V \mathrm{G} 90$ & 1.0379483 & QC110 (MVAr) & 22.04558 \\
\hline PG32 & 30.000000 & PG92 & 30.001991 & $V G 32$ & 1.0160001 & $V G 91$ & 1.0382000 & $T 8$ (p.u.) & 1.00028 \\
\hline PG34 & 30.000000 & PG99 & 30.000181 & $V G 34$ & 1.0352050 & $V G 92$ & 1.0402867 & $T 32$ (p.u.) & 1.07619 \\
\hline PG36 & 30.001076 & $P$ G100 & 176.002340 & $V G 36$ & 1.0296750 & $V G 99$ & 1.0306176 & $T 36$ (p.u.) & 1.00364 \\
\hline PG40 & 30.000031 & PG103 & 42.011701 & $V \mathrm{G} 40$ & 1.0235645 & $V G 100$ & 1.0330692 & $T 51$ (p.u.) & 0.99335 \\
\hline PG42 & 30.000005 & PG104 & 30.013010 & $V G 42$ & 1.0262225 & $V G 103$ & 1.0268264 & $T 93$ (p.u.) & 0.98511 \\
\hline PG46 & 35.710740 & PG105 & 30.000286 & $V \mathrm{G} 46$ & 1.0490460 & $V G 104$ & 1.0225026 & $T 95$ (p.u.) & 0.99528 \\
\hline PG49 & 161.740580 & $P$ G107 & 30.010348 & $V G 49$ & 1.0599033 & $V G 105$ & 1.0195927 & $T 102$ (p.u.) & 0.98316 \\
\hline PG54 & 44.400379 & PG110 & 30.000001 & $V G 54$ & 1.0437102 & $V G 107$ & 1.0125182 & $T 107$ (p.u.) & 0.94765 \\
\hline PG55 & 30.000000 & PG111 & 40.800000 & $V G 55$ & 1.0418340 & $V G 110$ & 1.0208466 & $T 127$ (p.u.) & 1.00670 \\
\hline PG56 & 30.000132 & PG112 & 30.000000 & $V G 56$ & 1.0426048 & $V G 111$ & 1.0285442 & Fuel cost $(\$ / \mathrm{h})$ & 134993.01000 \\
\hline PG59 & 125.766500 & PG113 & 30.014962 & $V$ G59 & 1.0587616 & $V G 112$ & 1.0118602 & Ploss (MW) & 59.44586 \\
\hline PG61 & 122.169800 & PG116 & 30.000000 & $V G 61$ & 1.0611259 & $V G 113$ & 1.0286230 & VD (p.u.) & 1.93219 \\
\hline PG62 & 30.000000 & & & VG62 & 1.0581949 & $V$ G116 & 1.0520616 & PG69 (MW) & 371.06085 \\
\hline
\end{tabular}


Table 21: Simulation results obtained from MODE-OPF algorithm for case 2 of IEEE 118-bus system.

\begin{tabular}{|c|c|c|c|c|c|c|c|c|c|}
\hline $\begin{array}{c}\text { Control } \\
\text { variables }\end{array}$ & Values (MW) & $\begin{array}{c}\text { Control } \\
\text { variables }\end{array}$ & Values (MW) & $\begin{array}{c}\text { Control } \\
\text { variables }\end{array}$ & Values (p.u.) & $\begin{array}{c}\text { Control } \\
\text { variables }\end{array}$ & Values (p.u.) & $\begin{array}{c}\text { Control } \\
\text { variables }\end{array}$ & Values \\
\hline PG1 (MW) & 73.720742 & PG65 & 147.300420 & VG1 (MW) & 1.026833 & $V \mathrm{G} 65$ & 1.049689 & QC5 (MVAr) & 18.048479 \\
\hline PG4 & 30.000000 & PG66 & 147.600000 & $V \mathrm{G} 4$ & 1.040823 & $V G 66$ & 1.029436 & QC34 (MVAr) & 0.038566 \\
\hline PG6 & 30.004433 & $P G 70$ & 30.000029 & VG6 & 1.037155 & $V G 69$ & 1.030806 & QC37 (MVAr) & 0.000000 \\
\hline PG8 & 30.001938 & $P G 72$ & 30.000750 & $V \mathrm{G} 8$ & 1.039729 & $V \mathrm{G} 70$ & 1.030839 & QC44 (MVAr) & 3.169625 \\
\hline$P \mathrm{G} 10$ & 165.032690 & $P G 73$ & 30.026081 & $V \mathrm{G} 10$ & 1.042859 & $V \mathrm{G} 72$ & 1.040309 & QC45 (MVAr) & 20.742639 \\
\hline$P \mathrm{G} 12$ & 133.414890 & $P$ G74 & 99.999670 & $V \mathrm{G} 12$ & 1.036036 & $V G 73$ & 1.037170 & QC46 (MVAr) & 10.750599 \\
\hline$P \mathrm{G} 15$ & 84.750920 & PG76 & 99.999999 & $V G 15$ & 1.029931 & $V \mathrm{G} 74$ & 1.029268 & QC48 (MVAr) & 8.594440 \\
\hline$P \mathrm{G} 18$ & 30.186756 & PG77 & 99.999907 & $V G 18$ & 1.029775 & $V G 76$ & 1.019345 & QC74 (MVAr) & 19.112107 \\
\hline$P \mathrm{G} 19$ & 57.238556 & PG80 & 288.217930 & $V G 19$ & 1.030083 & VG77 & 1.031227 & QC79 (MVAr) & 24.997969 \\
\hline$P \mathrm{G} 24$ & 30.003725 & PG85 & 30.000003 & $V \mathrm{G} 24$ & 1.039307 & $V G 80$ & 1.036393 & QC82 (MVAr) & 23.818645 \\
\hline$P G 25$ & 96.000000 & PG87 & 31.200000 & $V G 25$ & 1.043698 & $V G 85$ & 1.034078 & QC83 (MVAr) & 11.785550 \\
\hline$P$ G26 & 124.216760 & PG89 & 212.115160 & $V G 26$ & 1.058324 & VG87 & 1.052456 & $Q \mathrm{C} 105$ (MVAr) & 20.354596 \\
\hline$P$ G27 & 49.164404 & $P \mathrm{G} 90$ & 99.138561 & $V G 27$ & 1.030552 & $V G 89$ & 1.037422 & $Q \mathrm{C} 107$ (MVAr) & 16.786494 \\
\hline PG31 & 60.211705 & PG91 & 30.009251 & $V \mathrm{G} 31$ & 1.031086 & $V \mathrm{G} 90$ & 1.028969 & $Q \mathrm{C} 110$ (MVAr) & 15.138179 \\
\hline PG32 & 40.793179 & PG92 & 30.006111 & $V \mathrm{G} 32$ & 1.030975 & $V G 91$ & 1.031686 & $T 8$ (p.u.) & 0.998960 \\
\hline PG34 & 68.497437 & PG99 & 39.114352 & $V \mathrm{G} 34$ & 1.032924 & $V \mathrm{G} 92$ & 1.028674 & $T 32$ (p.u.) & 1.074251 \\
\hline PG36 & 52.253846 & $P G 100$ & 105.738610 & $V G 36$ & 1.030032 & $V G 99$ & 1.032062 & $T 36$ (p.u.) & 1.003425 \\
\hline$P \mathrm{G} 40$ & 100.000000 & $P G 103$ & 42.000207 & $V \mathrm{G} 40$ & 1.029303 & $V \mathrm{G} 100$ & 1.031176 & $T 51$ (p.u.) & 0.998566 \\
\hline$P \mathrm{G} 42$ & 99.975331 & PG104 & 33.044689 & $V G 42$ & 1.027713 & $V \mathrm{G} 103$ & 1.032144 & $T 93$ (p.u.) & 1.019130 \\
\hline$P \mathrm{G} 46$ & 82.433708 & $P G 105$ & 52.434424 & $V \mathrm{G} 46$ & 1.027924 & $V G 104$ & 1.029039 & $T 95$ (p.u.) & 1.018448 \\
\hline PG49 & 141.274360 & PG107 & 57.344047 & $V \mathrm{G} 49$ & 1.027232 & $V G 105$ & 1.028834 & $T 102$ (p.u.) & 0.993092 \\
\hline PG54 & 141.222870 & $P G 110$ & 30.000035 & $V$ G54 & 1.024479 & $V \mathrm{G} 107$ & 1.028575 & $T 107$ (p.u.) & 0.983730 \\
\hline PG55 & 77.869852 & $P G 111$ & 40.800233 & $V G 55$ & 1.024688 & $V \mathrm{G} 110$ & 1.031654 & $T 127$ (p.u.) & 0.996027 \\
\hline$P$ G56 & 99.999893 & $P G 112$ & 51.477770 & $V G 56$ & 1.024303 & $V G 111$ & 1.041056 & Ploss (MW) & 16.875146 \\
\hline PG59 & 253.224830 & PG113 & 30.000512 & $V G 59$ & 1.022357 & $V G 112$ & 1.027672 & Fuel cost $(\$ / \mathrm{h})$ & 155257.680000 \\
\hline$P$ G61 & 78.000661 & PG116 & 74.571457 & $V \mathrm{G} 61$ & 1.024260 & $V \mathrm{G} 113$ & 1.036436 & VD (p.u.) & 1.719105 \\
\hline$P$ G62 & 64.841019 & & & $V G 62$ & 1.023360 & $V G 116$ & 1.047035 & PG69 (MW) & 2.400438 \\
\hline
\end{tabular}

The performance of the proposed MODE-OPF has been compared to EJADE-SP, $\mathrm{C}^{2} \mathrm{oDE}$, LSHADE-44, UDE and ECHT-DE, while GA-MPC and FRORI have been excluded from the comparison as they were not able to produce feasible solutions for the IEEE 118-bus system. Table 22 presents the summary of the results obtained from MODEOPF and the competing algorithms for case1. The proposed MODE-OPF obtains the best results for best, median, mean, worst and standard deviation. Using MODE-OPF, a saving of 277.500\$, 4222.100\$, 3352.800\$, 42.511\$ and 13409.200\$ per hour is obtained in comparison with EJADE-SP, C ${ }^{2}$ oDE, LSHADE-44, UDE and ECHT-DE, respectively. Table 23 presents the the results obtained from the Wilcoxon signed sum ranked test. From this table, it is clear that the proposed algorithm is statistically better than all the rival algorithms.

Table 22: Case1: Comparative summary of algorithms for IEEE 118-bus system

\begin{tabular}{cccccc}
\hline Algorithms & Best & Median & Mean & Worst & Std. \\
\hline EJADE-SP & 135270.500 & 145634.000 & 144609.717 & 147032.900 & 2950.158 \\
C $^{2}$ oDE & 139215.100 & 140617.800 & 140563.803 & 142153.500 & 791.440 \\
LSHADE-44 & 138345.800 & 141460.000 & 141616.190 & 147084.200 & 1742.387 \\
UDE & 135035.511 & 135407.198 & 135556.783 & 136697.621 & 448.622 \\
ECHT-DE & 148402.200 & 156220.300 & 156511.647 & 169173.900 & 4641.558 \\
MODE-OPF & $\mathbf{1 3 4 9 9 3 . 0 0 0}$ & $\mathbf{1 3 5 0 6 8 . 4 5 0}$ & $\mathbf{1 3 5 0 7 7 . 5 6 7}$ & $\mathbf{1 3 5 1 8 6 . 0 0 0}$ & $\mathbf{4 6 . 9 2 6}$ \\
\hline
\end{tabular}

\begin{tabular}{|c|c|c|c|c|}
\hline Algorithms & $R^{+}$ & $R^{-}$ & Exact P-value & Dec. \\
\hline MODE-OPF vs. EJADE-SP & 465.0 & 0.0 & $1.8626 \mathrm{E}-9$ & + \\
\hline MODE-OPF vs. C $C^{2}$ oDE & 465.0 & 0.0 & $1.8626 \mathrm{E}-9$ & + \\
\hline MODE-OPF vs. LSHADE-44 & 465.0 & 0.0 & $1.8626 \mathrm{E}-9$ & + \\
\hline MODE-OPF vs. UDE & 456.0 & 9.0 & $6.146 \mathrm{E}-8$ & + \\
\hline MODE-OPF vs. ECHT-DE & 465.0 & 0.0 & $1.8626 \mathrm{E}-9$ & + \\
\hline
\end{tabular}

For minimizing the real power loss (case 2), the summary of the results obtained from the proposed MODE-OPF, 
EJADE-SP, C $^{2}$ oDE, LSHADE-44, UDE and ECHT-DE when solving the IEEE 118-bus system are presented in Table 24. The minimum power loss obtained by MODE-OPF, Considering the best results obtained MODE-OPF, EJADESP, $C^{2}$ oDE, LSHADE-44, UDE and ECHT-DE are 16.875150MW, 17.129120MW, 21.764760MW, 22.603160MW, 17.182191MW and $64.612650 \mathrm{MW}$, respectively. It is clear that, the proposed MODE-OPF obtains the minimum value for the real power loss among all the competing algorithms. Table 25 presents the summary od the results obtained from the Wilcoxon signed ranked test, from which the proposed MODE-OPF is statistically superior to all other algorithms.

Table 24: Case2: Comparative summary of algorithms for IEEE 118-bus system

\begin{tabular}{cccccc}
\hline Algorithms & Best & Median & Mean & Worst & Std. \\
\hline EJADE-SP & 17.129120 & 35.316460 & 17.631485 & 18.323512 & 3.240519 \\
C $^{2}$ ODE & 21.764760 & 29.442960 & 25.208170 & 25.420595 & 2.001475 \\
LSHADE-44 & 22.603160 & 48.072960 & 26.838230 & 27.669944 & 4.453681 \\
UDE & 17.182191 & 21.995836 & 17.873803 & 18.391651 & 1.274370 \\
ECHT-DE & 64.612650 & 99.516740 & 80.695495 & 81.569335 & 9.123206 \\
MODE-OPF & $\mathbf{1 6 . 8 7 5 1 5 0}$ & $\mathbf{1 7 . 8 5 6 3 7 0}$ & $\mathbf{1 7 . 3 2 9 3 7 0}$ & $\mathbf{1 7 . 3 4 1 6 4 5}$ & $\mathbf{0 . 2 5 2 5 4 7}$ \\
\hline
\end{tabular}

\begin{tabular}{|c|c|c|c|c|}
\hline Algorithms & $R^{+}$ & $R^{-}$ & Exact P-value & Dec. \\
\hline MODE-OPF vs. EJADE-SP & 406.0 & 59.0 & $1.5288 \mathrm{E}-4$ & + \\
\hline MODE-OPF vs. C ${ }^{2}$ oDE & 465.0 & 0.0 & $1.8626 \mathrm{E}-9$ & + \\
\hline MODE-OPF vs. LSHADE-44 & 465.0 & 0.0 & $1.8626 \mathrm{E}-9$ & + \\
\hline MODE-OPF vs. UDE & 436.0 & 29.0 & $3.24 \mathrm{E}-6$ & + \\
\hline MODE-OPF vs. ECHT-DE & 465.0 & 0.0 & $1.8626 \mathrm{E}-9$ & + \\
\hline
\end{tabular}

\section{Conclusion and Future Work}

In this paper, an enhanced multi-operator DE algorithm has been developed to solve the OPF problem. The proposed MODE-OPF utilizes the benefits of using multiple DE operators in a single algorithmic setting, with the most appropriate one emphasized based on both population diversity and quality of solutions. Also, to balance between diversification and fast convergence, an adaptive method (AM) has been developed. The proposed algorithm is tested by solving the OPF problem with the modified IEEE-30 bus system, where solar and wind generators are modeled to ensure that the OPF problem incorporates current issues of the power industry. Five different cases are disjointly considered with five unique objective functions. The performance of the proposed algorithm is evaluated against wellperformed algorithms. It is observed from the experimental results that the proposed MODE-OPF outperforms the other advanced algorithms to optimize the various objective functions. In terms of the quality of solutions, MODEOPF is able to decrease the total generation cost up to $24.08 \%$, the real power loss up to $6.80 \%$ and the total generation cost with emission up to $8.56 \%$. In terms of time, the proposed MODE-OPF was able to reduce the computational time up to $25.57 \%, 30.88 \%, 49.4 \%, 37.45 \%$ and $34.64 \%$ for Case 1, Case 2, Case 3, Case 4 and Case 5, respectively. To further demonstrate the effectiveness of the proposed algorithm, we have solved the OPF problem of IEEE-118 bus system. The results from the analysis again indicate the superiority of the algorithm.

In future, more constraint handling techniques will be considered with the proposed algorithm. The proposed MODE-OPF will also be tested with other power system problems, such as stochastic optimal power flow problem, optimal sizing of distributed generation for active power loss minimization and wind farm layout problem.

\section{Conflict of Interest:}

All the authors declare that they have no conflict of interest. 


\section{References}

[1] M. A. Hossain, H. R. Pota, M. J. Hossain, F. Blaabjerg, Evolution of microgrids with converter-interfaced generations: Challenges and opportunities, International Journal of Electrical Power \& Energy Systems 109 (2019) 160-186.

[2] M. A. Hossain, H. R. Pota, S. Squartini, A. F. Abdou, Modified PSO algorithm for real-time energy management in grid-connected microgrids, Renewable energy 136 (2019) 746-757.

[3] N. Daryani, M. T. Hagh, S. Teimourzadeh, Adaptive group search optimization algorithm for multi-objective optimal power flow problem, Applied Soft Computing 38 (2016) 1012-1024.

[4] J. Carpentier, Contribution to the economic dispatch problem, Bulletin de la Societe Francoise des Electriciens 3 (8) (1962) $431-447$.

[5] M. Abido, Optimal power flow using particle swarm optimization, International Journal of Electrical Power \& Energy Systems 24 (7) (2002) 563-571.

[6] B. C. Lesieutre, I. A. Hiskens, Convexity of the set of feasible injections and revenue adequacy in FTR markets, IEEE Transactions on Power Systems 20 (4) (2005) 1790-1798.

[7] T. A. Al-Muhawesh, I. S. Qamber, The established mega watt linear programming-based optimal power flow model applied to the real power 56-bus system in eastern province of Saudi Arabia, Energy 33 (1) (2008) 12-21.

[8] H. Habibollahzadeh, G.-X. Luo, A. Semlyen, Hydrothermal optimal power flow based on a combined linear and nonlinear programming methodology, IEEE Transactions on Power Systems 4 (2) (1989) 530-537.

[9] R. Burchett, H. Happ, D. Vierath, Quadratically convergent optimal power flow, IEEE Transactions on Power Apparatus and Systems (11) (1984) 3267-3275.

[10] X. Yan, V. H. Quintana, Improving an interior-point-based OPF by dynamic adjustments of step sizes and tolerances, IEEE Transactions on Power Systems 14 (2) (1999) 709-717.

[11] A. J. Santos, G. Da Costa, Optimal-power-flow solution by Newton's method applied to an augmented Lagrangian function, IEE ProceedingsGeneration, Transmission and Distribution 142 (1) (1995) 33-36.

[12] S. Li, W. Gong, L. Wang, X. Yan, C. Hu, Optimal power flow by means of improved adaptive differential evolution, Energy (2020) 117314.

[13] S. Duman, U. Güvenç, Y. Sönmez, N. Yörükeren, Optimal power flow using gravitational search algorithm, Energy Conversion and Management 59 (2012) 86-95.

[14] A. G. Bakirtzis, P. N. Biskas, C. E. Zoumas, V. Petridis, Optimal power flow by enhanced genetic algorithm, IEEE Transactions on power Systems 17 (2) (2002) 229-236.

[15] H. Bouchekara, Optimal power flow using black-hole-based optimization approach, Applied Soft Computing 24 (2014) 879-888.

[16] W. Bai, I. Eke, K. Y. Lee, An improved artificial bee colony optimization algorithm based on orthogonal learning for optimal power flow problem, Control Engineering Practice 61 (2017) 163-172.

[17] J. Radosavljević, D. Klimenta, M. Jevtić, N. Arsić, Optimal power flow using a hybrid optimization algorithm of particle swarm optimization and gravitational search algorithm, Electric Power Components and Systems 43 (17) (2015) 1958-1970.

[18] H. R. Bouchekara, A. Chaib, M. A. Abido, R. A. El-Sehiemy, Optimal power flow using an Improved Colliding Bodies Optimization algorithm, Applied Soft Computing 42 (2016) 119-131.

[19] A.-F. Attia, R. A. El Sehiemy, H. M. Hasanien, Optimal power flow solution in power systems using a novel Sine-Cosine algorithm, International Journal of Electrical Power \& Energy Systems 99 (2018) 331-343.

[20] A.-A. A. Mohamed, Y. S. Mohamed, A. A. El-Gaafary, A. M. Hemeida, Optimal power flow using moth swarm algorithm, Electric Power Systems Research 142 (2017) 190-206.

[21] T. T. Nguyen, A high performance social spider optimization algorithm for optimal power flow solution with single objective optimization, Energy 171 (2019) 218-240.

[22] C. Shilaja, T. Arunprasath, Optimal power flow using Moth Swarm Algorithm with Gravitational Search Algorithm considering wind power, Future Generation Computer Systems 98 (2019) 708-715.

[23] R. Storn, K. Price, Differential evolution-a simple and efficient heuristic for global optimization over continuous spaces, Journal of global optimization 11 (4) (1997) 341-359.

[24] S. Das, S. S. Mullick, P. N. Suganthan, Recent advances in differential evolution-an updated survey, Swarm and Evolutionary Computation 27 (2016) 1-30.

[25] J. Zhang, S. Lin, H. Liu, Y. Chen, M. Zhu, Y. Xu, A small-population based parallel differential evolution algorithm for short-term hydrothermal scheduling problem considering power flow constraints, Energy 123 (2017) 538-554.

[26] P. P. Biswas, P. N. Suganthan, R. Mallipeddi, G. A. Amaratunga, Optimal power flow solutions using differential evolution algorithm integrated with effective constraint handling techniques, Engineering Applications of Artificial Intelligence 68 (2018) 81-100.

[27] A. A. El-Fergany, H. M. Hasanien, Single and multi-objective optimal power flow using grey wolf optimizer and differential evolution algorithms, Electric Power Components and Systems 43 (13) (2015) 1548-1559.

[28] S. S. Reddy, Optimal power flow using hybrid differential evolution and harmony search algorithm, International Journal of Machine Learning and Cybernetics 10 (5) (2019) 1077-1091.

[29] K. M. Sallam, S. M. Elsayed, R. A. Sarker, D. L. Essam, Differential evolution with landscape-based operator selection for solving numerical optimization problems, in: Intelligent and evolutionary systems, Springer, 371-387, 2017.

[30] K. M. Sallam, S. M. Elsayed, R. A. Sarker, D. L. Essam, Improved United Multi-Operator Algorithm for Solving Optimization Problems, in: 2018 IEEE Congress on Evolutionary Computation (CEC), IEEE, 1-8, 2018

[31] K. M. Sallam, S. M. Elsayed, R. A. Sarker, D. L. Essam, Landscape-assisted multi-operator differential evolution for solving constrained optimization problems, Expert Systems with Applications (2019) 113033.

[32] S. M. Elsayed, R. A. Sarker, D. L. Essam, Multi-operator based evolutionary algorithms for solving constrained optimization problems, Computers \& operations research 38 (12) (2011) 1877-1896.

[33] S. Elsayed, R. Sarker, C. C. Coello, Enhanced multi-operator differential evolution for constrained optimization, in: 2016 IEEE Congress on Evolutionary Computation (CEC), IEEE, 4191-4198, 2016. 
[34] A. A. Eladl, A. A. ElDesouky, Optimal economic dispatch for multi heat-electric energy source power system, International Journal of Electrical Power \& Energy Systems 110 (2019) 21-35.

[35] S. S. Reddy, P. Bijwe, Real time economic dispatch considering renewable energy resources, Renewable Energy 83 (2015) 1215-1226.

[36] M. A. Hossain, H. R. Pota, S. Squartini, F. Zaman, J. M. Guerrero, Energy scheduling of community microgrid with battery cost using particle swarm optimisation, Applied Energy 254 (2019) 113723.

[37] T. P. Chang, Estimation of wind energy potential using different probability density functions, Applied Energy 88 (5) (2011) 1848-1856.

[38] M.-R. Chen, G.-Q. Zeng, K.-D. Lu, Constrained multi-objective population extremal optimization based economic-emission dispatch incorporating renewable energy resources, Renewable Energy 143 (2019) 277-294.

[39] P. P. Biswas, P. Suganthan, G. A. Amaratunga, Optimal power flow solutions incorporating stochastic wind and solar power, Energy Conversion and Management 148 (2017) 1194-1207.

[40] R.-H. Liang, J.-H. Liao, A fuzzy-optimization approach for generation scheduling with wind and solar energy systems, IEEE Transactions on Power Systems 22 (4) (2007) 1665-1674.

[41] F. L. Albuquerque, A. J. Moraes, G. C. Guimarães, S. M. Sanhueza, A. R. Vaz, Photovoltaic solar system connected to the electric power grid operating as active power generator and reactive power compensator, Solar Energy 84 (7) (2010) 1310-1317.

[42] N. Roy, H. Pota, M. Hossain, Reactive power management of distribution networks with wind generation for improving voltage stability, Renewable Energy 58 (2013) 85-94.

[43] K. M. Sallam, S. M. Elsayed, R. A. Sarker, D. L. Essam, Two-phase differential evolution framework for solving optimization problems, in: 2016 IEEE Symposium Series on Computational Intelligence (SSCI), IEEE, 1-8, 2016.

[44] R. Tanabe, A. S. Fukunaga, Improving the search performance of SHADE using linear population size reduction, in: 2014 IEEE congress on evolutionary computation (CEC), IEEE, 1658-1665, 2014

[45] K. M. Sallam, S. M. Elsayed, R. A. Sarker, D. L. Essam, Landscape-based adaptive operator selection mechanism for differential evolution, Information Sciences 418 (2017) 383-404.

[46] J. Zhang, A. C. Sanderson, JADE: adaptive differential evolution with optional external archive, IEEE Transactions on evolutionary computation 13 (5) (2009) 945-958.

[47] K. Deb, An efficient constraint handling method for genetic algorithms, Computer methods in applied mechanics and engineering 186 (2-4) (2000) 311-338.

[48] S. Elsayed, R. Sarker, C. C. Coello, T. Ray, Adaptation of operators and continuous control parameters in differential evolution for constrained optimization, Soft Computing 22 (19) (2018) 6595-6616.

[49] S. Duman, S. Rivera, J. Li, L. Wu, Optimal power flow of power systems with controllable wind-photovoltaic energy systems via differential evolutionary particle swarm optimization, International Transactions on Electrical Energy Systems 30 (4) (2020) e12270.

[50] L. Shi, C. Wang, L. Yao, Y. Ni, M. Bazargan, Optimal power flow solution incorporating wind power, IEEE Systems Journal 6 (2) (2011) 233-241.

[51] S. M. Elsayed, R. A. Sarker, D. L. Essam, GA with a new multi-parent crossover for solving IEEE-CEC2011 competition problems, in: 2011 IEEE congress of evolutionary computation (CEC), IEEE, 1034-1040, 2011.

[52] R. Mallipeddi, P. N. Suganthan, Ensemble of constraint handling techniques, IEEE Transactions on Evolutionary Computation 14 (4) (2010) 561-579.

[53] A. Trivedi, K. Sanyal, P. Verma, D. Srinivasan, A unified differential evolution algorithm for constrained optimization problems, in: 2017 IEEE Congress on Evolutionary Computation (CEC), IEEE, 1231-1238, 2017.

[54] R. Polakova, L-SHADE with competing strategies applied to constrained optimization, in: 2017 IEEE congress on evolutionary computation (CEC), IEEE, 1683-1689, 2017.

[55] B.-C. Wang, H.-X. Li, J.-P. Li, Y. Wang, Composite differential evolution for constrained evolutionary optimization, IEEE Transactions on Systems, Man, and Cybernetics: Systems 49 (7) (2018) 1482-1495.

[56] Y. Wang, B.-C. Wang, H.-X. Li, G. G. Yen, Incorporating objective function information into the feasibility rule for constrained evolutionary optimization, IEEE Transactions on Cybernetics 46 (12) (2015) 2938-2952.

[57] S. García, A. Fernández, J. Luengo, F. Herrera, Advanced nonparametric tests for multiple comparisons in the design of experiments in computational intelligence and data mining: Experimental analysis of power, Information Sciences 180 (10) (2010) 2044-2064.

[58] A. Chaib, H. Bouchekara, R. Mehasni, M. Abido, Optimal power flow with emission and non-smooth cost functions using backtracking search optimization algorithm, International Journal of Electrical Power \& Energy Systems 81 (2016) 64-77.

[59] O. Alsac, B. Stott, Optimal load flow with steady-state security, IEEE transactions on power apparatus and systems (3) (1974) 745-751. 